1 Hacettepe Journal of Mathematics and Statistics

$\bigcap$ Volume 48 (3) (2019), 897 -930

RESEARCH ARTICLE

\title{
Method for generating distributions and classes of probability distributions: the univariate case
}

\author{
Cícero Ramos de Brito* (1) , Leandro Chaves Rêgo ${ }^{\dagger}$ (D) , Wilson Rosa de Oliveira ${ }^{\ddagger}$ (D) and \\ Frank Gomes-Silva $\$$ I
}

\begin{abstract}
In this work, we present a method to generate probability distributions and classes of probability distributions, which broadens the process of probability distribution construction. In this method, distribution classes are built from predefined monotonic functions and from known distributions. With its use, we can obtain different classes of probability distributions described in literature. Beside these results, we obtain results on the support and nature of the generated distributions.
\end{abstract}

Keywords: Probabilistic distributions generating method, Distribution classes generating functions, probabilistic distributions classes, probabilistic distribution support.

Mathematics Subject Classification (2010): 60E05; 62N05.

Received : 15.12.2017 Accepted : 28.06.2018 Doi : 10.15672/HJMS.2018.619

*Federal Institute of Pernambuco, Recife, Brazil. Email: cicerocarlosbrito@yahoo.com.br

${ }^{\dagger}$ Department of Statistics and Applied Mathematics, Federal University of Ceará, Fortaleza, Brazil. Email: leandrochavesrego@gmail.com

${ }^{\ddagger}$ Department of Statistics and Informatics, Federal Rural University of Pernambuco, Recife, Brazil. Email: wilson. rosalgmail.com

$\S_{\text {Department }}$ of Statistics and Informatics, Federal Rural University of Pernambuco, Recife, Brazil. Email: franksinatrags@gmail.com

${ }^{\top}$ Corresponding Author. 


\section{Introduction}

The amount of data available for analysis is growing increasingly faster, requiring new probabilistic distributions to better describe each phenomenon or experiment studied. Computer based tools allow the use of more complex distributions with a larger number of parameters to better study sizeable masses of data.

The literature in the field describes several generalizations and extensions of symmetric, asymmetric, discrete and continuous distributions. It is worth quoting Lee et al. (2013) regarding the main methods of generating distributions and classes of probability distributions:

Generally speaking, the methods developed prior to 1980s may be summarized into three categories: (1) method of differential equation, (2) method of transformation, and (3) quantile method. Techniques developed since 1980s may be categorized as methods of combination for the reason that these methods attempt to combine existing distributions into new distributions or adding new parameters to an existing distribution. (Lee et al., 2013, 219)

The relevance of these new models is that, according to the situation, each one of them can better fit the mass of data. Table 1 presents several classes of distributions described in literature, their nomenclature and the title of the work where they have been presented. For a comprehensive discussion about the classes of probability distributions see three excellent articles written by Lee et al. (2013), Tahir and Nadarajah (2015) and Tahir and Cordeiro (2016).

Table 1. Some classes of distributions described in literatura

\begin{tabular}{|c|c|}
\hline Distribution classes & Nomenclature \\
\hline$F(x)=G^{a}(x)$, where $a>0$ & $\begin{array}{l}\text { defined by Mudholkar et al. } \\
\text { (1995) }\end{array}$ \\
\hline $\begin{array}{c}F(x)=\frac{1}{B(a, b)} \int_{0}^{G(x)} t^{a-1}(1-t)^{b-1} d t \\
\text { where } a>0, b>0 \text { and } 0<t<1\end{array}$ & $\begin{array}{l}\text { beta- } G \text { type } 1 \text { defined by } \\
\text { Eugene et al. (2002) }\end{array}$ \\
\hline $\begin{array}{c}F(x)=\frac{1}{B(a, b)} \int_{0}^{G(x)} t^{a-1}(1+t)^{-(a+b)} d t, \\
\quad \text { where } a>0, b>0 \text { and } t>0\end{array}$ & $\begin{array}{l}\text { beta- } G \text { type } 3 \text { defined by } \\
\text { Thair and Nadarajah }(2015)\end{array}$ \\
\hline $\begin{array}{c}F(x)=\frac{1}{B(a, b)} \int_{0}^{G^{c}(x)} t^{a-1}(1-t)^{b-1} d t \\
\text { where } a>0, b>0, c>0 \text { and } 0<t<1\end{array}$ & $\begin{array}{l}\text { Mc- } G \text { type } 1 \text { defined by } \\
\text { McDonald (1984) }\end{array}$ \\
\hline $\begin{array}{c}F(x)=\frac{1}{B(a, b)} \int_{0}^{G^{c}(x)} t^{a-1}(1+t)^{-(a+b)} d t, \\
\quad \text { where } a>0, b>0, c>0 \text { and } t>0\end{array}$ & $\begin{array}{l}\text { Mc- } G \text { type } 3 \text { defined by } \\
\text { Thair and Nadarajah }(2015)\end{array}$ \\
\hline $\begin{aligned} F(x)= & 1-\left(1-G^{a}(x)\right)^{b}, \text { where } \\
& a>0 \text { and } b>0\end{aligned}$ & $\begin{array}{l}\text { Kumaraswamy- } G \text { defined by } \\
\text { Cordeiro and Castro (2011) }\end{array}$ \\
\hline $\begin{array}{c}F(x)=1-\left(1-(1-G(x))^{a}\right)^{b}, \text { where } \\
a>0 \text { and } b>0\end{array}$ & $\begin{array}{l}\text { Kumaraswamy- } G \text { type } 2 \\
\text { defined by } \\
\text { Thair and Nadarajah (2015) }\end{array}$ \\
\hline$F(x)=1-\frac{G(x)}{G(x)+b(1-G(x)}$, where $b>0$ & $\begin{array}{l}\text { Marshall-Olkin- } G \text { defined by } \\
\text { Marshall and Olkin (1997) }\end{array}$ \\
\hline $\begin{array}{c}F(x)=1-\left(\frac{b(1-G(x))}{G(x)+b(1-G(x)}\right)^{\theta}, \text { where } b>0 \text { and } \\
\theta>0\end{array}$ & $\begin{array}{l}\text { Marshall-Olkin- } G \\
\text { defined by Jayakumar } \\
\text { and Mathew (2008) }\end{array}$ \\
\hline$F(x)=\left(\frac{G(x)}{G(x)+b(1-G(x)}\right)^{\theta}$, where $b>0$ and $\theta>0$ & $\begin{array}{l}\text { Marshall-Olkin- } G \text { defined by } \\
\text { Thair and Nadarajah (2015) }\end{array}$ \\
\hline & gamma- $G$ defined by \\
\hline
\end{tabular}


Table 1. Continued from the previous page

\begin{tabular}{|c|c|}
\hline Distribution classes & Nomenclature \\
\hline $\begin{array}{c}F(x)=\frac{\beta^{\alpha}}{\Gamma(\alpha)} \int_{0}^{-\log [1-G(x)]} t^{\alpha-1} \mathrm{e}^{-\beta t} d t, \\
\text { where } \alpha>0 \text { and } \beta>0\end{array}$ & $\begin{array}{l}\text { Zografos and Balakrishnan } \\
\text { (2009) }\end{array}$ \\
\hline $\begin{array}{c}F(x)=1-\frac{\beta^{\alpha}}{\Gamma(\alpha)} \int_{0}^{\frac{1-G(x)}{G(x)}} t^{\alpha-1} \mathrm{e}^{-\beta t} d t, \\
\text { where } \alpha>0 \text { and } \beta>0\end{array}$ & $\begin{array}{l}\text { gamma- } G \text { defined by } \\
\text { Brito et al. (2017) }\end{array}$ \\
\hline $\begin{array}{c}F(x)=1-\frac{\beta^{\alpha}}{\Gamma(\alpha)} \int_{0}^{-\log (G(x))} t^{\alpha-1} \mathrm{e}^{-\beta t} d t \\
\text { where } \alpha>0 \text { and } \beta>0\end{array}$ & $\begin{array}{l}\text { gamma- } G \text { defined by } \\
\text { Cordeiro et al. (2017) }\end{array}$ \\
\hline $\begin{array}{c}F(x)=1-\frac{a+[1-G(x)]}{(1+a)[1-G(x)]} \exp \left\{-a \frac{G(x)}{1-G(x)}\right\} \\
\text { where } a>0\end{array}$ & $\begin{array}{l}\text { Odd Lindley- } G \text { defined by } \\
\text { Gomes-Silva et al. (2017) }\end{array}$ \\
\hline $\begin{array}{c}F(x)=1-\frac{C\left(\theta \mathrm{e}^{-\alpha H(x)}\right)}{C(\theta)} \text {, where } x>0, \theta>0 \text { and } \\
C(\theta)=\sum_{n=1}^{\infty} a_{n} \theta^{n}\end{array}$ & $\begin{array}{l}\text { Extended Weibull distribution } \\
\text { defined by Silva et al. (2013) }\end{array}$ \\
\hline$F(x)=\frac{1-\exp [-\lambda G(x)]}{1-\mathrm{e}^{-\lambda}}$ & $\begin{array}{l}\text { Kumaraswamy- } G \text { Poisson defi- } \\
\text { ned by Ramos et al. (2014) }\end{array}$ \\
\hline $\begin{array}{c}F(x)=\left\{1-\left[1-G^{a}(x)\right]^{b}\right\}^{c}, \text { where } a>0, b>0 \\
\text { and } c>0\end{array}$ & $\begin{array}{l}\text { exponentiated } \\
\text { Kumaraswamy- } G \text { defined by } \\
\text { Silva (2019) }\end{array}$ \\
\hline$F(x)=\frac{\mathrm{e}^{\lambda \mathrm{e}^{-\beta x^{a}}}-\mathrm{e}^{\lambda}}{1-\mathrm{e}^{\lambda}}, x>0$ & $\begin{array}{l}\text { beta Weibull Poisson Family } \\
\text { defined by Percontini (2013) }\end{array}$ \\
\hline $\begin{array}{c}F(x)=\int_{0}^{G(x)} K t^{a-1}(1-t)^{b-1} \exp (-c t) d t, \text { where } \\
a>0, b>0 \text { and } c \in \mathbb{R}\end{array}$ & $\begin{array}{l}\text { beta Kummer generalized } \\
\text { defined by Pescim et al. } \\
\text { (2012) }\end{array}$ \\
\hline $\begin{array}{c}F(x)=\frac{\mathrm{e}^{-\frac{\lambda}{a} W\left(-a \mathrm{e}^{-a}\right)}-\mathrm{e}^{-\frac{\lambda}{a} W(\psi(x))}}{\mathrm{e}^{-\frac{\lambda}{a} W\left(-a \mathrm{e}^{-a}\right)}}, \text { where } \\
W(x)=\sum_{n=1}^{\infty} \frac{(-1)^{n-1} n^{n-z}}{(n-1) !} x^{n} \text { and } \\
\psi(x)=-a \mathrm{e}^{-a-b x^{a}}\end{array}$ & $\begin{array}{l}\text { Weibull Generalized Poisson } \\
\text { distribution defined } \\
\text { by Percontini (2014) }\end{array}$ \\
\hline$F(x)=\frac{(1-\beta)^{s}-\{1-\beta[1-G(x)]\}^{-s}}{(1-\beta)^{s}-1}$, & $\begin{array}{l}G \text {-Negative Binomial family } \\
\text { defined by Percontini (2014) }\end{array}$ \\
\hline $\begin{array}{c}F(x)=\frac{\zeta(s)-L i_{s}[1-G(x)]}{(s)}, \text { where } \\
L i_{s}(z)=\sum_{k=1}^{\infty} \frac{z^{k}}{k^{s}} \text { and } \zeta(s)=\sum_{k=1}^{\infty} \frac{1}{k^{s}}\end{array}$ & $\begin{array}{l}\text { Zeta- } G \text { defined by Percontini } \\
\text { (2014) }\end{array}$ \\
\hline$F(x)=\sum_{k=0}^{x} \frac{C^{(k)}(a)}{k ! C(\lambda)}(\lambda-a)^{k}$ & $\begin{array}{l}\text { Power Series Distributions } \\
\text { Family by Consul and Famoye, } \\
\text { (2006) }\end{array}$ \\
\hline$F(x)=\sum_{k=1}^{1} \frac{1}{k !}\left[(C(0))^{k}\right]^{(k-1)}$ & $\begin{array}{l}\text { Basic Lagrangian defined by } \\
\text { Consul and Famoye (2006) }\end{array}$ \\
\hline$F(x)=\sum_{k=n}^{x} \frac{n}{(k-n) ! k}\left[(C(0))^{k}\right]^{(k-n)}$ & Lagrangian Delta defined by \\
\hline
\end{tabular}


Table 1. Continued from the previous page

\begin{tabular}{|c|c|}
\hline Distribution classes & Nomenclature \\
\hline & Consul and Famoye (2006) \\
\hline $\begin{array}{c}F(x)=\left(\sum_{k=0}^{x} P(X=k)\right)^{\delta}, \\
w(0), \\
P(X=k)=\left\{\begin{array}{cc}k=0 \\
{\left[(C(0))^{k} w^{(1)}(0)\right]^{(k-1)}, k=1,2, \ldots}\end{array}\right.\end{array}$ & $\begin{array}{l}\text { Generalized Lagrangian } \\
\text { defined by Consul and } \\
\text { Famoye (2006) }\end{array}$ \\
\hline$F(x)=\int_{-\infty}^{x} \mathrm{e}^{\int \frac{a_{0}+a_{1} t+\cdots+a_{s} t^{s}}{b_{0}+b_{1} t+\cdots+b_{r} t^{r}} d t} d t$ & $\begin{array}{l}\text { Generalized Pearson in } \\
\text { Ordinary Differential } \\
\text { Equation form defined by } \\
\text { Shakil et al. (2010) }\end{array}$ \\
\hline$F(x)=\int_{-\infty}^{x} \mathrm{e}^{\int \frac{a_{0}+a_{1} t+\cdots+a_{s} t^{s}}{b_{0}+b_{1} t+\cdots+b_{r} t^{r}}(f(t))^{\beta}} d t d t, \quad \beta \geq 0$ & $\begin{array}{l}\text { Generalized Pearson in } \\
\text { Ordinary Differential } \\
\text { Equation form defined by } \\
\text { Shakil et al. (2010) }\end{array}$ \\
\hline$F(x)=\int_{-\infty}^{x} \int_{-\infty}^{y}\left(\sum_{i=1}^{2} a_{i}(t) f^{\beta_{i}}(t)\right) d t d y$ & $\begin{array}{l}\text { Generalized Family in } \\
\text { Ordinary Differential } \\
\text { Equation form defined by } \\
\text { Voda (2009) }\end{array}$ \\
\hline $\begin{array}{c}F(x)=\int_{a}^{W[G(x)]} r(t) d t \\
\text { where } W[G(x)] \in[a, b], \text { with } \\
W[G(x)] \text { differentiable and monotonically } \\
\text { non-decreasing }\end{array}$ & $\begin{array}{l}\text { T-X class } \\
\text { by Alzaatreh et al. (2013) }\end{array}$ \\
\hline
\end{tabular}

The aim of this work is to propose a method to create distributions and probabilistic distribution classes that could unify the various methods to generate distribution classes already described in literature. The idea of this method is to generate classes from already known distributions, using monotonic functions and a cumulative distribution function.

We show that the proposed method has high power of generality. The well-known T-X class generalizes most of the classes presented in Table 1. To get an idea of its power we will show that the T-X class appears as a sub-case of a simple sub-model of the proposed method that we will denote it by 3S1C1.2 (see Table 2, page 907). In addition to generalizing existing classes, the new method provides a source of new probability distribution classes.

This paper is organized in the following way: in Section 2, we describe two methods to generate probability distributions, establishing the conditions that must be satisfied by the used monotonic functions and probability distribution to guarantee that the proposed method indeed generates a probability distribution. In Section 3, we analyze a special case of the methods described in the previous section for the case where the monotonic functions are compositions of known probability distribution functions. Still in Section 3 we present several specific cases of these methods that may be easily used to obtain new probability distributions. At the end of this section, we demonstrate that all methods presented in Sections 2 and 3 are equivalent. In Section 4, we analyze the support and nature of the distributions generated by the methods proposed in Section 3. Section 5 
presents our conclusions and directions for further works. As an application of the proposed methods, the appendix A to the article contains a table (Table 4) showing how to obtain several classes of probability distributions described in the literature using our proposed methods.

\section{The method}

The method we suggest to create distribution classes uses monotonic functions, $U: \mathbb{R} \rightarrow \mathbb{R}$, $\sqrt{ }: \mathbb{R} \rightarrow \mathbb{R}, U_{j}: \mathbb{R} \rightarrow \mathbb{R} \cup\{ \pm \infty\}, L_{j}: \mathbb{R} \rightarrow \mathbb{R} \cup\{ \pm \infty\}, M_{j}: \mathbb{R} \rightarrow \mathbb{R} \cup\{ \pm \infty\}$ and $V_{j}: \mathbb{R} \rightarrow \mathbb{R} \cup\{ \pm \infty\}$, and a cumulative distribution function $(c d f) F$. The idea of this method is to generate a probability distribution integrating $F$ from $L_{j}(x)$ to $U_{j}(x)$ and from $M_{j}(x)$ to $V_{j}(x)$ for any $x \in \mathbb{R}$ and $j=1,2,3, \ldots, n$. Theorem 1 that follows shows sufficient conditions that the functions $V(x), \sqrt{ }(x), L_{j}(x), U_{j}(x), M_{j}(x)$ and $V_{j}(x)$ must satisfy to guarantee that the method generates a probability distribution function.

1. Theorem (T1). Method to generate distributions and classes of probability distributions.

Let $F: \mathbb{R} \rightarrow \mathbb{R}, \mathcal{V}: \mathbb{R} \rightarrow \mathbb{R}, \sqrt{ }: \mathbb{R} \rightarrow \mathbb{R}, U_{j}: \mathbb{R} \rightarrow \mathbb{R} \cup\{ \pm \infty\}, L_{j}: \mathbb{R} \rightarrow \mathbb{R} \cup\{ \pm \infty\}$, $M_{j}: \mathbb{R} \rightarrow \mathbb{R} \cup\{ \pm \infty\}$ and $V_{j}: \mathbb{R} \rightarrow \mathbb{R} \cup\{ \pm \infty\}$, for $j=1,2,3, \ldots, n$, be monotonic and right continuous functions such that:

[c1] $F$ is a cdf and $U$ and $\sqrt{ }$ are non-negative;

$[c 2] \mathcal{V}(x), U_{j}(x)$ and $M_{j}(x)$ are non-decreasing and $\sqrt{ }(x), V_{j}(x)$ and $L_{j}(x)$ are non-increasing $\forall j=1,2,3, \ldots, n$;

[c3] If $\lim _{x \rightarrow-\infty} U(x) \neq \lim _{x \rightarrow-\infty} \sqrt{ }(x)$, then $\lim _{x \rightarrow-\infty} V(x)=0$ or $\lim _{x \rightarrow-\infty} U_{j}(x)=\lim _{x \rightarrow-\infty} L_{j}(x) \forall$ $j=1,2,3, \ldots, n$, and $\lim _{x \rightarrow-\infty} \sqrt{ }(x)=0$ or $\lim _{x \rightarrow-\infty} M_{j}(x)=\lim _{x \rightarrow-\infty} V_{j}(x), \forall j=1,2,3, \ldots, n$;

[c4] If $\lim _{x \rightarrow-\infty} \mathcal{V}(x)=\lim _{x \rightarrow-\infty} \sqrt{ }(x) \neq 0$, then $\lim _{x \rightarrow-\infty} U_{j}(x)=\lim _{x \rightarrow-\infty} V_{j}(x)$ and $\lim _{x \rightarrow-\infty} M_{j}(x)=$ $\lim _{x \rightarrow-\infty} L_{j}(x), \forall j=1,2,3, \ldots, n$;

[c5] $\lim _{x \rightarrow-\infty} L_{j}(x) \leq \lim _{x \rightarrow-\infty} U_{j}(x)$ and if $\lim _{x \rightarrow-\infty} \sqrt{ }(x) \neq 0$, then $\lim _{x \rightarrow+\infty} M_{j}(x) \leq \lim _{x \rightarrow+\infty} V_{j}(x)$, $\forall j=1,2,3, \ldots, n$;

$[c 6] \lim _{x \rightarrow+\infty} U_{n}(x) \geq \sup \{x \in \mathbb{R}: F(x)<1\}$ and $\lim _{x \rightarrow+\infty} L_{1}(x) \leq \inf \{x \in \mathbb{R}: F(x)>0\}$;

$[c 7] \lim _{x \rightarrow+\infty} \mathcal{V}(x)=1$;

$[c 8] \lim _{x \rightarrow+\infty} \sqrt{ }(x)=0$ or $\lim _{x \rightarrow+\infty} M_{j}(x)=\lim _{x \rightarrow+\infty} V_{j}(x), \forall j=1,2,3, \ldots, n$ and $n \geq 1$;

$[c 9] \lim _{x \rightarrow+\infty} U_{j}(x)=\lim _{x \rightarrow+\infty} L_{j}+1(x), \forall j=1,2,3, \ldots, n-1$ and $n \geq 2$;

[c10] $F$ is a cdf without points of discontinuity or all functions $L_{j}(x)$ and $V_{j}(x)$ are constant at the right of the vicinity of points whose image are points of discontinuity of $F$, being also continuous in that points. Moreover, $F$ does not have any point of discontinuity in the set $\left\{\lim _{x \rightarrow \pm \infty} L_{j}(x)\right.$,

$\lim _{x \rightarrow \pm \infty} U_{j}(x), \lim _{x \rightarrow \pm \infty} M_{j}(x), \lim _{x \rightarrow \pm \infty} V_{j}(x)$, for some $\left.j=1,2, \ldots, n\right\}$. Then,

$$
H(x)=v(x) \sum_{j=1}^{n} \int_{L_{j}(x)}^{U_{j}(x)} d F(t)-\sqrt{ }(x) \sum_{j=1}^{n} \int_{M_{j}(x)}^{V_{j}(x)} d F(t)
$$

is a cdf. 
Proof. (i) $\lim _{x \rightarrow-\infty} H(x)=0$.

$$
\begin{aligned}
& \lim _{x \rightarrow-\infty} H(x)=\lim _{x \rightarrow-\infty}\left(V(x) \sum_{j=1}^{n} \int_{L_{j}(x)}^{U_{j}(x)} d F(t)\right) \\
& \text { - } \lim _{x \rightarrow-\infty}\left(\sqrt{ }(x) \sum_{j=1}^{n} \int_{M_{j}(x)}^{V_{j}(x)} d F(t)\right) \\
& =\left(\lim _{x \rightarrow-\infty} V(x)\right) \sum_{j=1}^{n} \int_{x \rightarrow-\infty} \lim _{x \rightarrow-\infty} U_{j}(x) d F(t) \\
& \text { - }\left(\lim _{x \rightarrow-\infty} \sqrt{ }(x)\right) \sum_{j=1}^{n} \int_{x \rightarrow-\infty} \lim _{x \rightarrow-\infty} V_{j}(x) d F(t),
\end{aligned}
$$

where the last equality holds because $F$ is continuous in

$$
\left\{\lim _{x \rightarrow-\infty} U_{j}(x), \lim _{x \rightarrow-\infty} L_{j}(x), \lim _{x \rightarrow-\infty} V_{j}(x), \lim _{x \rightarrow-\infty} M_{j}(x)\right\} .
$$

Conditions [c3] and [c4] guarantee that:

$$
\begin{aligned}
\lim _{x \rightarrow-\infty} H(x) & =\left(\lim _{x \rightarrow-\infty} V(x)\right) \sum_{j=1}^{n} \int_{x \rightarrow-\infty} \lim _{x \rightarrow-\infty} L_{j}(x) \\
& -\left(\lim _{x \rightarrow-\infty} \sqrt{ }(x)\right) \sum_{j=1}^{n} \int_{x \rightarrow-\infty} \lim _{x \rightarrow-\infty} V_{j}(x) \\
\lim _{j}(x) & d F(t)=0 .
\end{aligned}
$$

(ii) $\lim _{x \rightarrow+\infty} H(x)=1$.

$$
\begin{aligned}
\lim _{x \rightarrow+\infty} H(x) & =\lim _{x \rightarrow+\infty}\left(V(x) \sum_{j=1}^{n} \int_{L_{j}(x)}^{U_{j}(x)} d F(t)\right) \\
& -\lim _{x \rightarrow+\infty}\left(\sqrt{ }(x) \sum_{j=1}^{n} \int_{M_{j}(x)}^{V_{j}(x)} d F(t)\right) \\
& =\left(\lim _{x \rightarrow+\infty} V(x)\right) \sum_{j=1}^{n} \int_{x \rightarrow+\infty}^{\lim _{x \rightarrow+\infty} L_{j}(x)} d F(t) \\
& -\left(\lim _{x \rightarrow+\infty} \sqrt{ }(x)\right) \sum_{j=1}^{n} \int_{x \rightarrow+\infty}^{\lim _{x \rightarrow+\infty} V_{j}(x)} d F(t),
\end{aligned}
$$

where the last equality holds because $F$ is continuous in

$$
\left\{\lim _{x \rightarrow+\infty} U_{j}(x), \lim _{x \rightarrow+\infty} L_{j}(x), \lim _{x \rightarrow+\infty} V_{j}(x), \lim _{x \rightarrow+\infty} M_{j}(x)\right\} .
$$

Thus, conditions [c1], [c6], [c7], [c8] and [c9] guarantee that

$$
\lim _{x \rightarrow+\infty} H(x)=1 \text {. }
$$

(iii) If $x_{1} \leq x_{2}$, then $H\left(x_{1}\right) \leq H\left(x_{2}\right)$.

Let $x_{1} \leq x_{2}$, then [c2] implies that: $U_{j}\left(x_{1}\right) \leq U_{j}\left(x_{2}\right), L_{j}\left(x_{1}\right) \geq L_{j}\left(x_{2}\right), M_{j}\left(x_{1}\right) \leq$ $M_{j}\left(x_{2}\right), V_{j}\left(x_{1}\right) \geq V_{j}\left(x_{2}\right), V\left(x_{1}\right) \leq V\left(x_{2}\right)$ and $\sqrt{ }\left(x_{1}\right) \geq \sqrt{ }\left(x_{2}\right)$. Beside this, [c2] and [c5] imply, $\sum_{j=1}^{n} \int_{L_{j}\left(x_{1}\right)}^{U_{j}\left(x_{1}\right)} d F(t) \geq 0, \sum_{j=1}^{n} \int_{M_{j}\left(x_{1}\right)}^{V_{j}\left(x_{1}\right)} d F(t) \geq 0, \sum_{j=1}^{n} \int_{L_{j}\left(x_{2}\right)}^{U_{j}\left(x_{2}\right)} d F(t) \geq 0$ and $\sum_{j=1}^{n} \int_{M_{j}\left(x_{2}\right)}^{V_{j}\left(x_{2}\right)} d F(t) \geq 0$. 
Thus, since, by [c1], $V$ and $\sqrt{ }$ are non-negative, we have

$$
\begin{aligned}
H\left(x_{1}\right) & =V\left(x_{1}\right) \sum_{j=1}^{n} \int_{L_{j}\left(x_{1}\right)}^{U_{j}\left(x_{1}\right)} d F(t)-\sqrt{ }\left(x_{1}\right) \sum_{j=1}^{n} \int_{M_{j}\left(x_{1}\right)}^{V_{j}\left(x_{1}\right)} d F(t) \\
\leq & V\left(x_{2}\right) \sum_{j=1}^{n} \int_{L_{j}\left(x_{2}\right)}^{U_{j}\left(x_{2}\right)} d F(t)-\sqrt{ }\left(x_{2}\right) \sum_{j=1}^{n} \int_{M_{j}\left(x_{2}\right)}^{V_{j}\left(x_{2}\right)} d F(t)=H\left(x_{2}\right) .
\end{aligned}
$$

(iv) $\lim _{x \rightarrow x_{0}^{+}} H(x)=H\left(x_{0}\right)$.

$$
\begin{aligned}
& \lim _{x \rightarrow x_{0}^{+}} H(x)=\lim _{x \rightarrow x_{0}^{+}} V(x) \sum_{j=1}^{n} \int_{L_{j}(x)}^{U_{j}(x)} d F(t)-\lim _{x \rightarrow x_{0}^{+}} \sqrt{ }(x) \sum_{j=1}^{n} \int_{M_{j}(x)}^{V_{j}(x)} d F(t) \\
= & \left(\lim _{x \rightarrow x_{0}^{+}} V(x)\right) \sum_{j=1}^{n} \int_{\lim _{x \rightarrow x_{0}^{+}} L_{j}(x)}^{\lim _{j}(x)} d F(t)-\left(\lim _{x \rightarrow x_{0}^{+}} \sqrt{ }(x)\right) \sum_{j=1}^{n} \int_{\lim _{x \rightarrow x_{0}^{+}} M_{j}(x)}^{\lim _{j}(x)} d F(t) \\
= & V\left(x_{0}\right) \sum_{j=1}^{n} \int_{L_{j}\left(x_{0}\right)}^{U_{j}\left(x_{0}\right)} d F(t)-\sqrt{ }\left(x_{0}\right) \sum_{j=1}^{n} \int_{M_{j}\left(x_{0}\right)}^{V_{j}\left(x_{0}\right)} d F(t)=H\left(x_{0}\right) .
\end{aligned}
$$

The above equalities hold due to [c10] and because $V(x), U_{j}(x), M_{j}(x), \sqrt{ }(x), V_{j}(x)$ and $L_{j}(x)$ are right continuous.

From the facts $(i),(i i),(i i i)$ and $(i v)$, we may conclude that (2.1) is a $c d f$.

Corollary 1.1 presents an alternative method to generate distributions and classes of probability distributions.

1.1. Corollary (C1.1). Complementary method to generate distributions and classes of probability distributions.

Let $\varphi: \mathbb{R} \rightarrow \mathbb{R}, \mho: \mathbb{R} \rightarrow \mathbb{R}, W: \mathbb{R} \rightarrow \mathbb{R}, \mathbb{U}: \mathbb{R} \rightarrow \mathbb{R} \cup\{ \pm \infty\}, \mathbb{M}_{j}: \mathbb{R} \rightarrow \mathbb{R} \cup\{ \pm \infty\}$ and $\mathbb{V}_{j}: \mathbb{R} \rightarrow \mathbb{R} \cup\{ \pm \infty\}, \forall j=1,2,3, \ldots, n$, be monotonic and right continuous functions such that:

[cc1] $F$ is a cdf and $\mho$ and $W$ are non-negative;

$[c c 2] \mho(x), \mathbb{U}_{j}(x)$ and $\mathbb{M}_{j}(x)$ are non-decreasing and $W(x), \mathbb{V}_{j}(x)$ and $\mathbb{L}_{j}(x)$ are non-increasing $\forall j=1,2,3, \ldots, n$;

$$
\begin{aligned}
& \text { [cc3] If } \lim _{x \rightarrow+\infty} W(x) \neq \lim _{x \rightarrow+\infty} \mho(x) \text {, then } \lim _{x \rightarrow+\infty} \mho(x)=0 \text { or } \lim _{x \rightarrow+\infty} \mathbb{L}_{j}(x)=\lim _{x \rightarrow+\infty} \\
& \mathbb{U}_{j}(x), \forall j=1,2,3, \ldots, n \text {, and } \lim _{x \rightarrow+\infty} W(x)=0 \text { or } \lim _{x \rightarrow+\infty} \mathbb{M}_{j}(x)=\lim _{x \rightarrow+\infty} \mathbb{V}_{j}(x), \forall \\
& j=1,2,3, \ldots, n \text {; } \\
& \text { [cc4] If } \lim _{x \rightarrow+\infty} W(x)=\lim _{x \rightarrow+\infty} \mho(x) \neq 0 \text {, then } \lim _{x \rightarrow+\infty} \mathbb{U}_{j}(x)=\lim _{x \rightarrow+\infty} \mathbb{V}_{j}(x) \text { and } \lim _{x \rightarrow+\infty} \\
& \mathbb{M}_{j}(x)=\lim _{x \rightarrow+\infty} \mathbb{L}_{j}(x), \forall j=1,2,3, \ldots, n ; \\
& {[c c 5] \lim _{x \rightarrow+\infty} \mathbb{M}_{j}(x) \leq \lim _{x \rightarrow+\infty} \mathbb{V}_{j}(x) \text { and if } \lim _{x \rightarrow+\infty} \mho(x) \neq 0 \text {, then } \lim _{x \rightarrow-\infty} \mathbb{L}_{j}(x) \leq \lim _{x \rightarrow-\infty} \mathbb{U}_{j}(x) \text {, }} \\
& \forall j=1,2,3, \ldots, n \text {; } \\
& {[c c 6] \lim _{x \rightarrow-\infty} \mathbb{V}_{n}(x) \geq \sup \{x \in \mathbb{R}: \varphi(x)<1\} \text { and } \lim _{x \rightarrow-\infty} L_{1}(x) \leq \inf \{x \in \mathbb{R}: \varphi(x)>0\} ;} \\
& \text { [cc7] } \lim _{x \rightarrow-\infty} W(x)=1 \\
& {[c c 8] \lim _{x \rightarrow-\infty} \mho(x)=0 \text { or } \lim _{x \rightarrow-\infty} \mathbb{L}_{j}(x)=\lim _{x \rightarrow-\infty} \mathbb{U}_{j}(x), \forall j=1,2,3, \ldots, n \text { and } n \geq 1 \text {; }}
\end{aligned}
$$


$[c c 9] \lim _{x \rightarrow-\infty} \mathbb{V}_{j}(x)=\lim _{x \rightarrow-\infty} \mathbb{M}_{j}+1(x), \forall j=1,2,3, \ldots, n-1$ and $n \geq 2 ;$

[cc10] $\varphi$ is a cdf without points of discontinuity or all functions $\mathbb{L}_{j}(x)$ and $\mathbb{V}_{j}(x)$ are constant at the right of the vicinity of points whose image are points of discontinuity of $\varphi$, being also continuous in that points. Moreover, $\varphi$ does not have any point of discontinuity in the set $\left\{\lim _{x \rightarrow \pm \infty} \mathbb{L}_{j}(x)(x)\right.$, $\lim _{x \rightarrow \pm \infty} \mathbb{U}_{j}(x), \lim _{x \rightarrow \pm \infty} \mathbb{M}_{j}(x), \lim _{x \rightarrow \pm \infty} \mathbb{V}_{j}(x)$, for some $\left.j=1,2, \ldots, n\right\}$.

Then,

$$
H(x)=1-W(x) \sum_{j=1}^{n} \int_{\mathbb{M}_{j}(x)}^{\mathbb{V}_{j}(x)} d \varphi(t)+\mho(x) \sum_{j=1}^{n} \int_{\mathbb{L}_{j}(x)}^{\mathbb{U}_{j}(x)} d \varphi(t)
$$

is a cdf.

Proof. In Theorem 1, consider $n=1, \mathcal{V}(x)=1, \sqrt{ }(x)=0, U_{1}(x)=1$ and $L_{1}(x)=$ $W(x) \sum_{j=1}^{n} \int_{\mathbb{M}_{j}(x)}^{\mathbb{V}_{j}(x)} d \varphi(t)-\mho(x) \sum_{j=1}^{n} \int_{\mathbb{L}_{j}(x)}^{\mathbb{U}_{j}(x)} d \varphi(t), \forall x \in \mathbb{R}$, and $F$ a $c d f$ of the uni-

form $[0,1]$ distribution. Note that $U_{1}(x)$ and $L_{1}(x)$ satisfy the hypotheses of Theorem 1 , since [cc1], [cc2] and [cc5] guarantee that $L_{1}(x)=W(x) \sum_{j=1}^{n} \int_{\mathbb{M}_{j}(x)}^{\mathbb{V}_{j}(x)} d \varphi(t)-\mho(x) \sum_{j=1}^{n} \int_{\mathbb{L}_{j}(x)}^{\mathbb{U}_{j}(x)} d \varphi(t)$ is non-increasing and $U_{1}(x)=1$ is non-decreasing. Thus, conditions [cc2] and [cc5] are satisfied. Moreover, conditions [cc3] and [cc4] guarantee that:

$\lim _{x \rightarrow-\infty} U_{1}(x)=\lim _{x \rightarrow-\infty} L_{1}(x)=1, \lim _{x \rightarrow+\infty} U_{1}(x)=\sup \{x \in \mathbb{R}: F(x)<1\}=1, \lim _{x \rightarrow+\infty} L_{1}(x)=$ $\inf \{x \in \mathbb{R}: F(x)>0\}=0$, that both $L_{1}(x)$ and $U_{1}(x)$ are right continuous and that $F$ is a $c d f$ without points of discontinuity.

As all conditions of Theorem 1 are satisfied, it follows that

$$
\begin{aligned}
& H(x)=\int_{L_{1}(x)}^{U_{1}(x)} d F(s)=\int_{W(x) \sum_{j=1}^{n} \int_{\mathbb{M}_{j}(x)}^{\mathbb{V}_{j}(x)} d \varphi(t)-\mho(x) \sum_{j=1}^{n} \int_{L_{j}(x)}^{U_{j}(x)} d \varphi(t)}^{1} d s \\
& =1-W(x) \sum_{j=1}^{n} \int_{\mathbb{M}_{j}(x)}^{\mathbb{V}_{j}(x)} d \varphi(t)+\mho(x) \sum_{j=1}^{n} \int_{\mathbb{L}_{j}(x)}^{\mathbb{U}_{j}(x)} d \varphi(t)
\end{aligned}
$$

is a $c d f$.

In the next section, we present some corollaries of Theorem 1 where the monotonic functions $V(x), \sqrt{ }(x), U_{j}(x), L_{j}(x), M_{j}(x)$ and $V_{j}(x)$ are compositions of monotonic functions of known probability distributions.

\section{Monotonic functions involving probabilities distributions}

In this section, we show how to generate classes of probability distributions using monotonic functions which are compositions known probability distributions. Formally, consider that $u$ : $[0,1]^{m} \rightarrow \mathbb{R}, \vartheta:[0,1]^{m} \rightarrow \mathbb{R}, \mu_{j}:[0,1]^{m} \rightarrow \mathbb{R} \cup\{ \pm \infty\}, \ell:[0,1]^{m} \rightarrow \mathbb{R} \cup\{ \pm \infty\}$, $v_{j}:[0,1]^{m} \rightarrow \mathbb{R} \cup\{ \pm \infty\}$ and $m_{j}:[0,1]^{m} \rightarrow \mathbb{R} \cup\{ \pm \infty\}$ are monotonic and right continuous functions. The results of this section are achieved considering that: $v(x)=U\left(G_{1}, \ldots, G_{m}\right)(x)$, $\sqrt{ }(x)=\vartheta\left(G_{1}, \ldots, G_{m}\right)(x), U_{j}(x)=\mu_{j}\left(G_{1}, \ldots, G_{m}\right)(x), L_{j}(x)=\ell\left(G_{1}, \ldots, G_{m}\right)(x), M_{j}$ $(x)=m_{j}\left(G_{1}, \ldots, G_{m}\right)(x)$ and $V_{j}(x)=v_{j}\left(G_{1}, \ldots, G_{m}\right)(x)$.

We use the abbreviation $(\cdot)(x)=\left(G_{1}, \ldots, G_{m}\right)(x)=\left(G_{1}(x), \ldots, G_{m}(x)\right)$ to represent the vector formed by the $c d f^{\prime} s$ calculated on the same point of the domain $x$. 
1.2. Corollary (C1.2). Method to generate classes of probability distributions.

Let $F: \mathbb{R} \rightarrow \mathbb{R}, \mu_{j}:[0,1]^{m} \rightarrow \mathbb{R} \cup\{ \pm \infty\}, \ell_{j}:[0,1]^{m} \rightarrow \mathbb{R} \cup\{ \pm \infty\}, u:[0,1]^{m} \rightarrow \mathbb{R}$, $v_{j}:[0,1]^{m} \rightarrow \mathbb{R} \cup\{ \pm \infty\}, m_{j}:[0,1]^{m} \rightarrow \mathbb{R} \cup\{ \pm \infty\}$ and $\vartheta:[0,1]^{m} \rightarrow \mathbb{R}, \forall j=1,2,3, \ldots, n$, be monotonic and right continuous functions such that:

$[d 1] F$ is a cdf and $U$ and $\vartheta$ are non-negative;

$[d 2] \mu_{j}, m_{j}$ and $U$ are non-decreasing and $\ell_{j}, v_{j}$ and $\vartheta$ are non-increasing, $\forall j=1,2,3, \ldots, n$, in all of its variables;

[d3] If $U(0, \ldots, 0) \neq \vartheta(0, \ldots, 0)$, then $u(0, \ldots, 0)=0$ or $\mu_{j}(0, \ldots, 0)=\ell_{j}(0, \ldots, 0), \forall$ $j=1,2,3, \ldots, n$, and $\vartheta(0, \ldots, 0)=0$ or $m_{j}(0, \ldots, 0)=v_{j}(0, \ldots, 0), \forall j=1,2,3, \ldots, n$;

[d4] If $U(0, \ldots, 0)=\vartheta(0, \ldots, 0) \neq 0$, then $\mu_{j}(0, \ldots, 0)=v_{j}(0, \ldots, 0)$ and $m_{j}(0, \ldots, 0)$

$=\ell_{j}(0, \ldots, 0), \forall j=1,2,3, \ldots, n$;

$[d 5] \ell_{j}(0, \ldots, 0) \leq \mu_{j}(0, \ldots, 0)$ and if $\vartheta(0, \ldots, 0) \neq 0$, then $m_{j}(1, \ldots, 1) \leq v_{j}(1, \ldots, 1)$, $\forall j=1,2,3, \ldots, n$;

$[d 6] \mu_{n}(1, \ldots, 1) \geq \sup \{x \in \mathbb{R}: F(x)<1\}$ and $\ell_{1}(1, \ldots, 1) \leq \inf \{x \in \mathbb{R}: F(x)>0\} ;$

$[d 7] U(1, \ldots, 1)=1$

$[d 8] \vartheta(1, \ldots, 1)=0$ or $v_{j}(1, \ldots, 1)=m_{j}(1, \ldots, 1), \forall j=1,2,3, \ldots, n-1$ and $n \geq 2$;

$[d 9] \mu_{j}(1, \ldots, 1)=\ell_{j+1}(1, \ldots, 1), \forall j=1,2,3, \ldots, n-1$ and $n \geq 2$;

[d10] $F$ is a cdf without points of discontinuity or the functions $\ell_{j}(\cdot)(x)$ and $v_{j}(\cdot)(x)$ are constant at the right of the vicinity of points whose image are points of discontinuity of $F$, being also continuous in that points. Moreover, $F$ does not have any point of discontinuity in the set $\left\{\ell_{j}(0, \ldots, 0)\right.$, $\mu_{j}(0, \ldots, 0), m_{j}(0, \ldots, 0), v_{j}(0, \ldots, 0), \ell_{j}(1, \ldots, 1), \mu_{j}(1, \ldots, 1), m_{j}(1, \ldots, 1), v_{j}(1, \ldots, 1)$, for some $j=1,2, \ldots, n\}$.

Then,

$$
H_{G_{1}, \ldots, G_{m}}(x)=\boldsymbol{u}(\cdot)(x) \sum_{j=1}^{n} \int_{\ell_{j}(\cdot)(x)}^{\mu_{j}(\cdot)(x)} d F(t)-\vartheta(\cdot)(x) \int_{m_{j}(\cdot)(x)}^{v_{j}(\cdot)(x)} d F(t)
$$

is a functional generator of classes of probability distributions where $(\cdot)(x)=\left(G_{1}, \ldots\right.$, $\left.G_{m}\right)(x)$.

Proof. In Theorem 1, set $V(x)=U(\cdot)(x), \sqrt{ }(x)=\vartheta(\cdot)(x), U_{j}(x)=\mu_{j}(\cdot)(x), L_{j}(x)=$ $\ell_{j}(\cdot)(x), M_{j}(x)=m_{j}(\cdot)(x)$ and $V_{j}(x)=v_{j}(\cdot)(x)$, and observe that condition $[d i]$ implies condition $[c i]$ of Theorem 1 , for $i=1,2, \ldots, 10$.

Let us now consider a special case of Corollary 1.2 that is a functional constructor of classes of probability distributions that can be easily used.

$1^{\text {st }}$ special case of Corollary 1.2 (1C1.2). Easy to use method for the construction of classes of probability distributions.

Let $u_{i}:[0,1]^{m} \rightarrow[0,1]$ and $\nu_{i}:[0,1]^{m} \rightarrow[0,1]$ be monotonic and right continuous functions such that $u_{i}$ 's are non-decreasing and $\nu_{i}$ 's are non-increasing in each one of its variables, with $u_{i}(0, \ldots, 0)=0, u_{i}(1, \ldots, 1)=1, \nu_{i}(0, \ldots, 0)=1$ and $\nu_{i}(1, \ldots, 1)=0$, for all 
$i=1, \ldots, k$. If, in Corollary 1.2, $u(\cdot)(x)=\prod_{i=1}^{k}\left(\left(i-\theta_{i}\right) u_{i}(\cdot)(x)+\theta_{i}\right)^{\alpha_{i}}$ and $\vartheta(\cdot)(x)=$ $\prod_{i=1}^{k}\left(\theta_{i} \nu_{i}(\cdot)(x)\right)^{\alpha_{i}}$, with $\alpha_{i} \geq 0$ and $0 \leq \theta_{i} \leq 1$, then

$$
\begin{aligned}
H_{G_{1}, \ldots, G_{m}}(x) & =\prod_{i=1}^{k}\left(\left(1-\theta_{i}\right) u_{i}(\cdot)(x)+\theta_{i}\right)^{\alpha_{i}} \sum_{j=1}^{n} \int_{\ell_{j}(\cdot)(x)}^{\mu_{j}(\cdot)(x)} d F(t) \\
& -\prod_{i=1}^{k}\left(\theta_{i} \nu_{i}(\cdot)(x)\right)^{\alpha_{i}} \sum_{j=1}^{n} \int_{m_{j}(\cdot)(x)}^{v_{j}(\cdot)(x)} d F(t),
\end{aligned}
$$

is a functional generator of classes of probability distributions, where $(\cdot)(x)=\left(G_{1}, \ldots\right.$, $\left.G_{m}\right)(x)$.

Table 2 shows some particular cases of the functional constructor of classes of probability distributions, given by Equation (3.1), that may be more easily used for the generation of classes of distribution. Consider the following functions in the expressions from 15S1C1.2 to 20S1C1.2: $\mu:[0,1] \rightarrow \mathbb{R} \cup\{ \pm \infty\}, \ell:[0,1] \rightarrow \mathbb{R} \cup\{ \pm \infty\}, v:[0,1] \rightarrow \mathbb{R} \cup\{ \pm \infty\}, m:[0,1] \rightarrow \mathbb{R} \cup\{ \pm \infty\}$ such that $\mu$ and $m$ are non-decreasing and right continuous, and $v$ and $\ell$ are non-increasing and right continuous. 


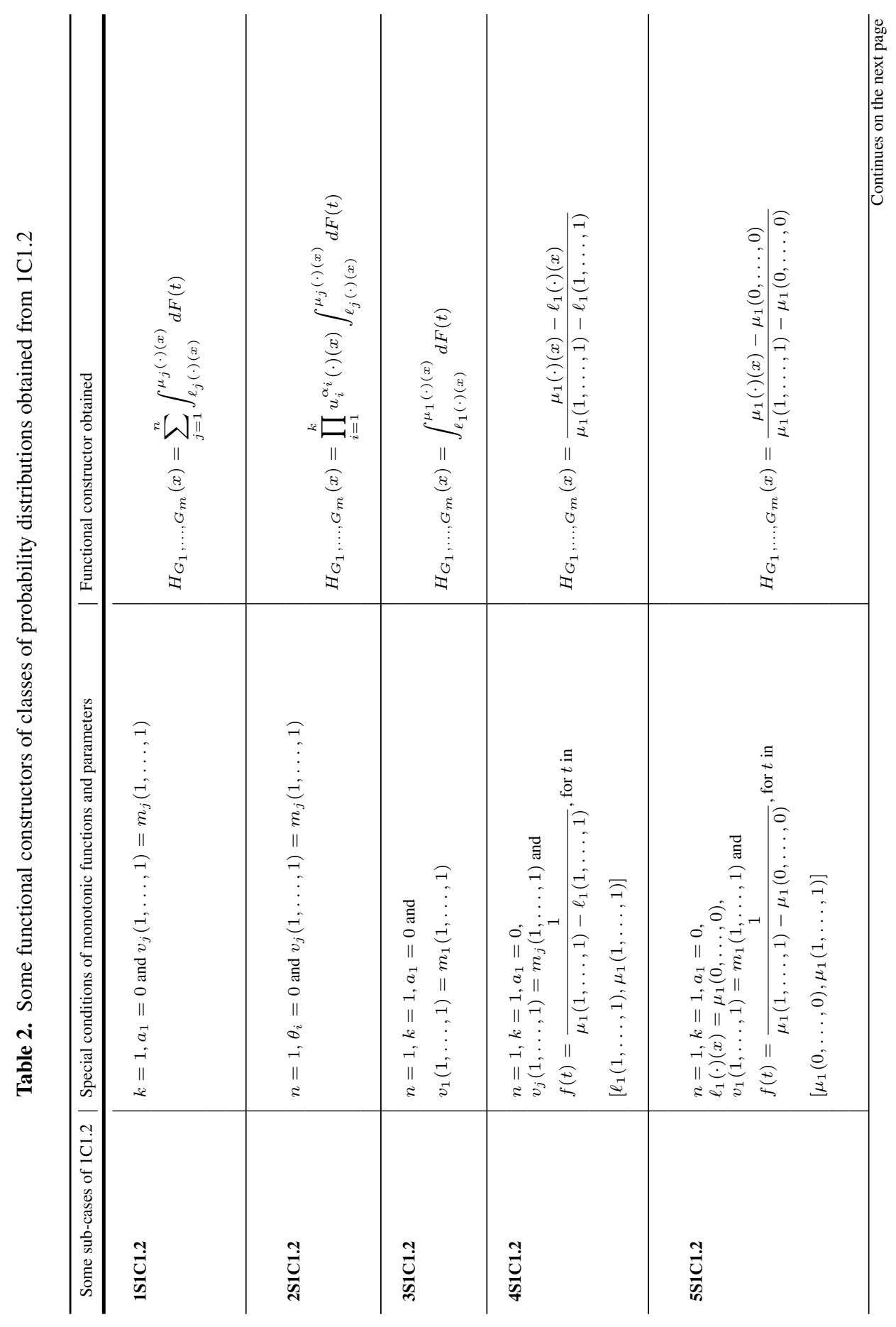




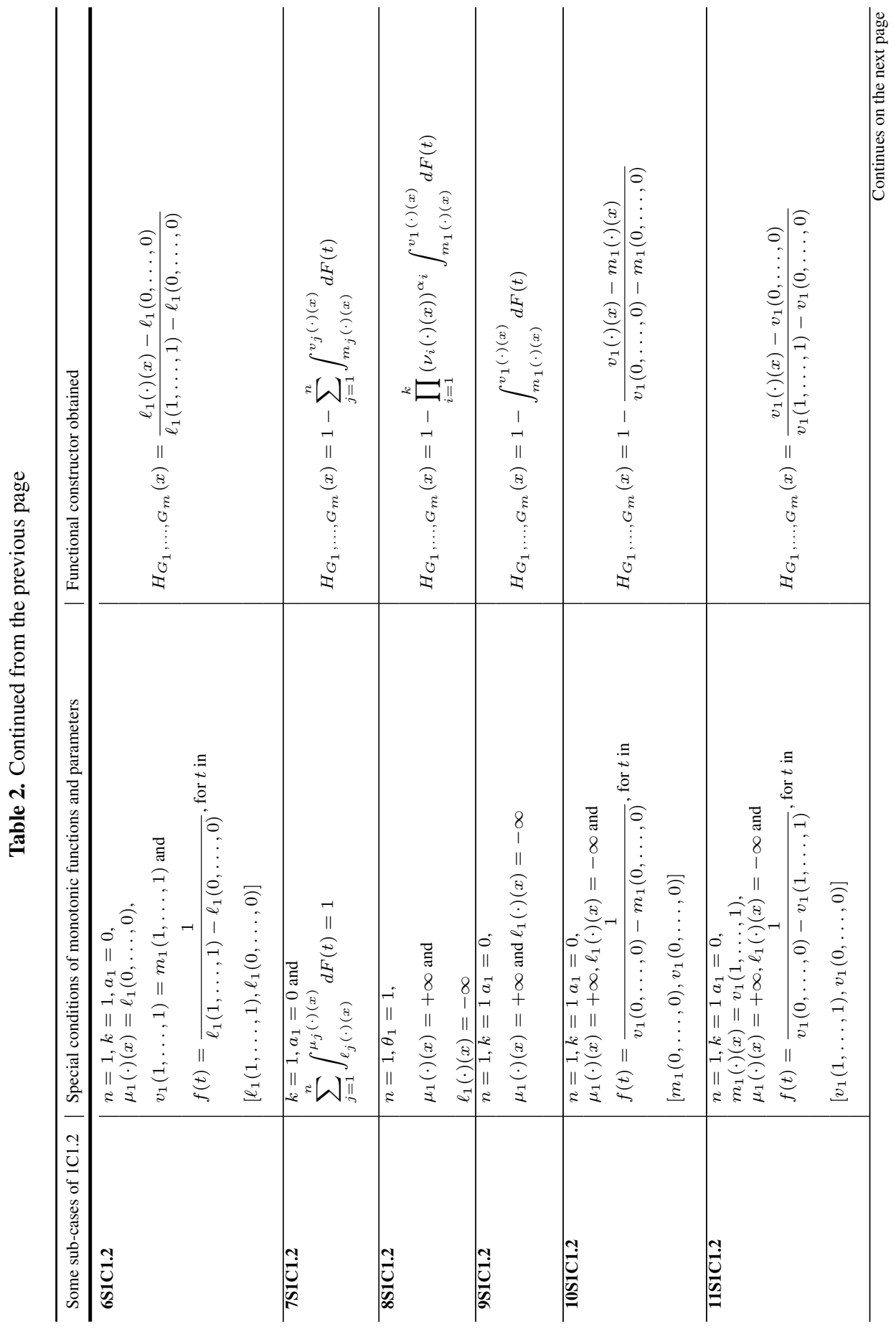




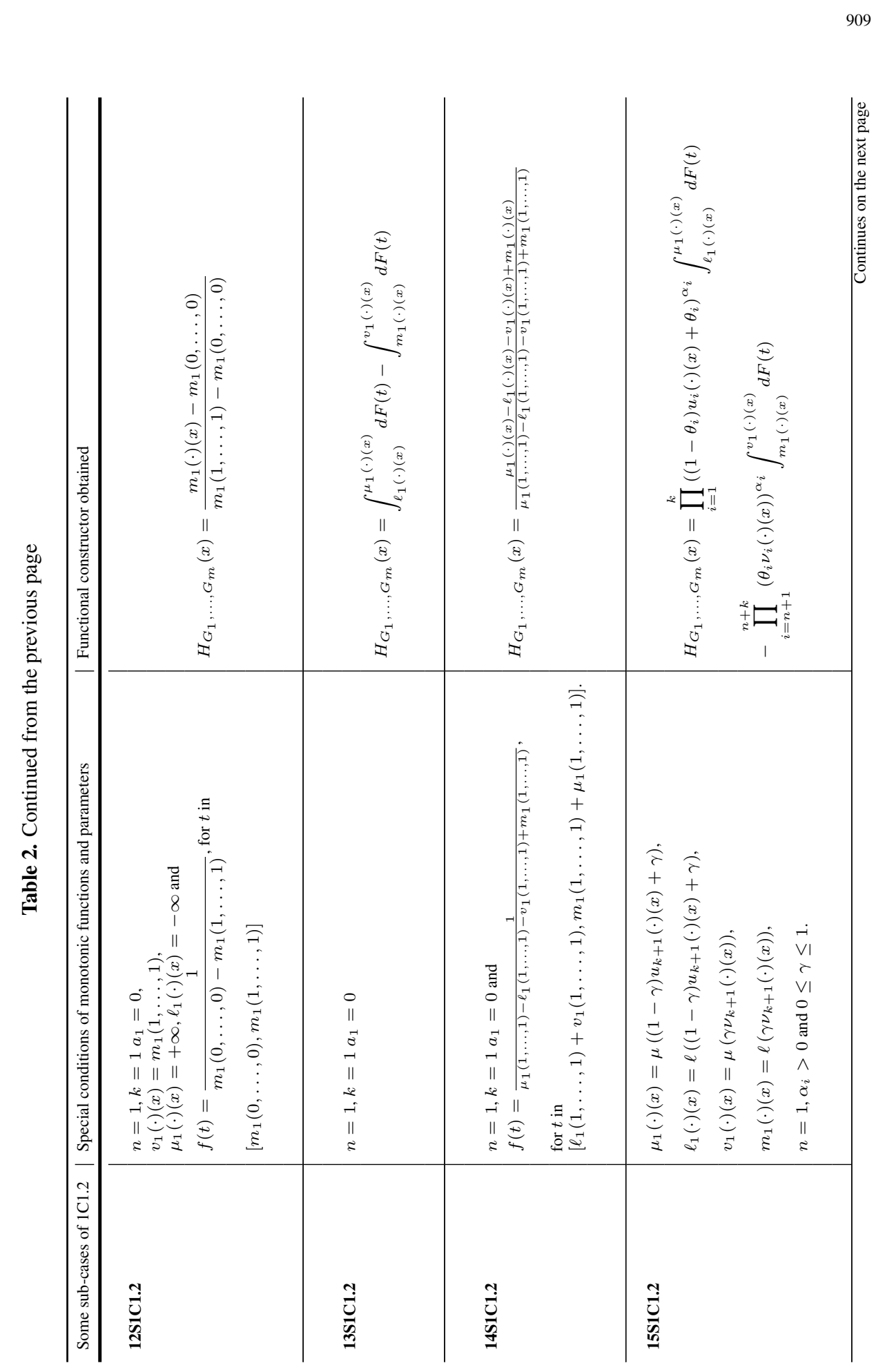




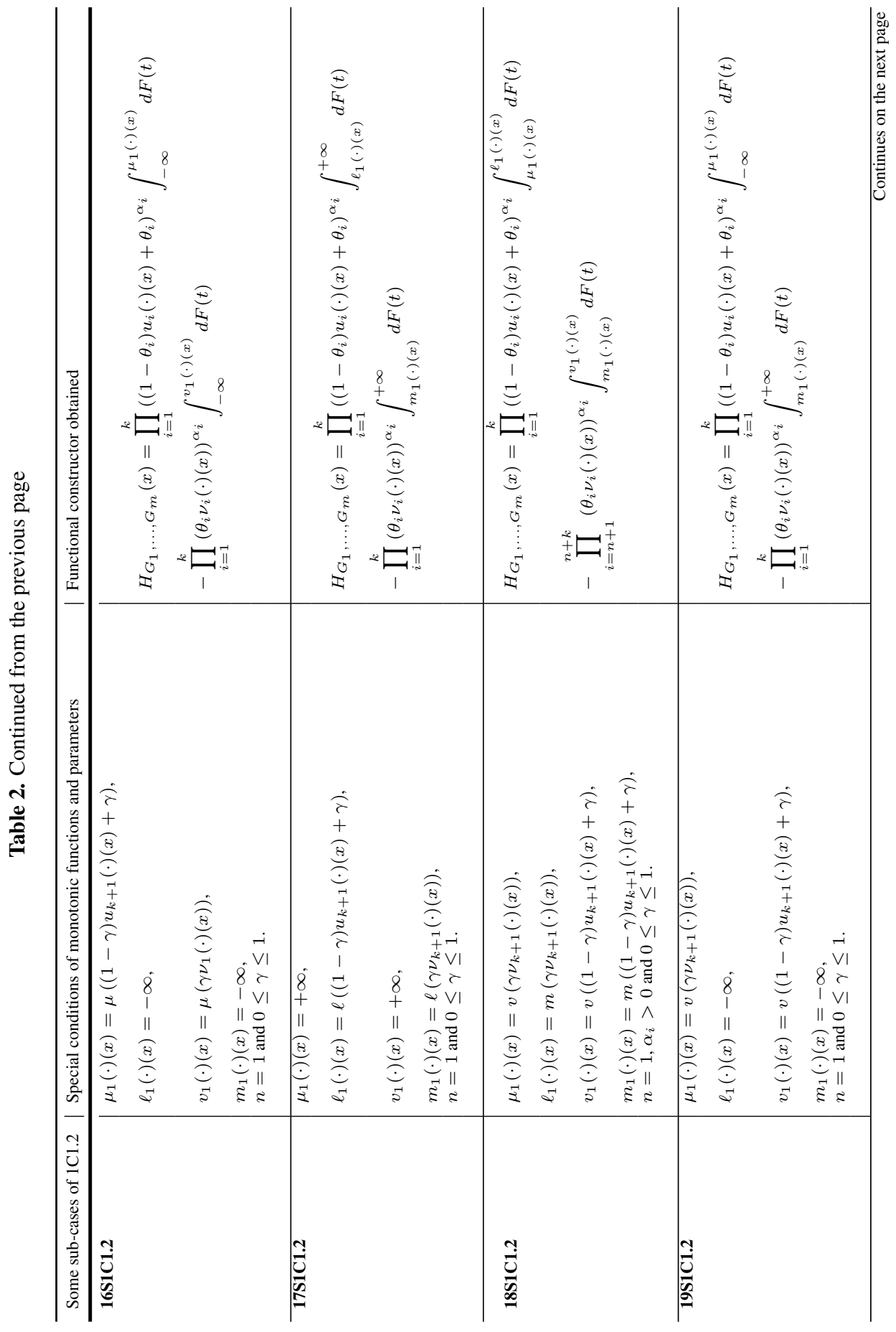




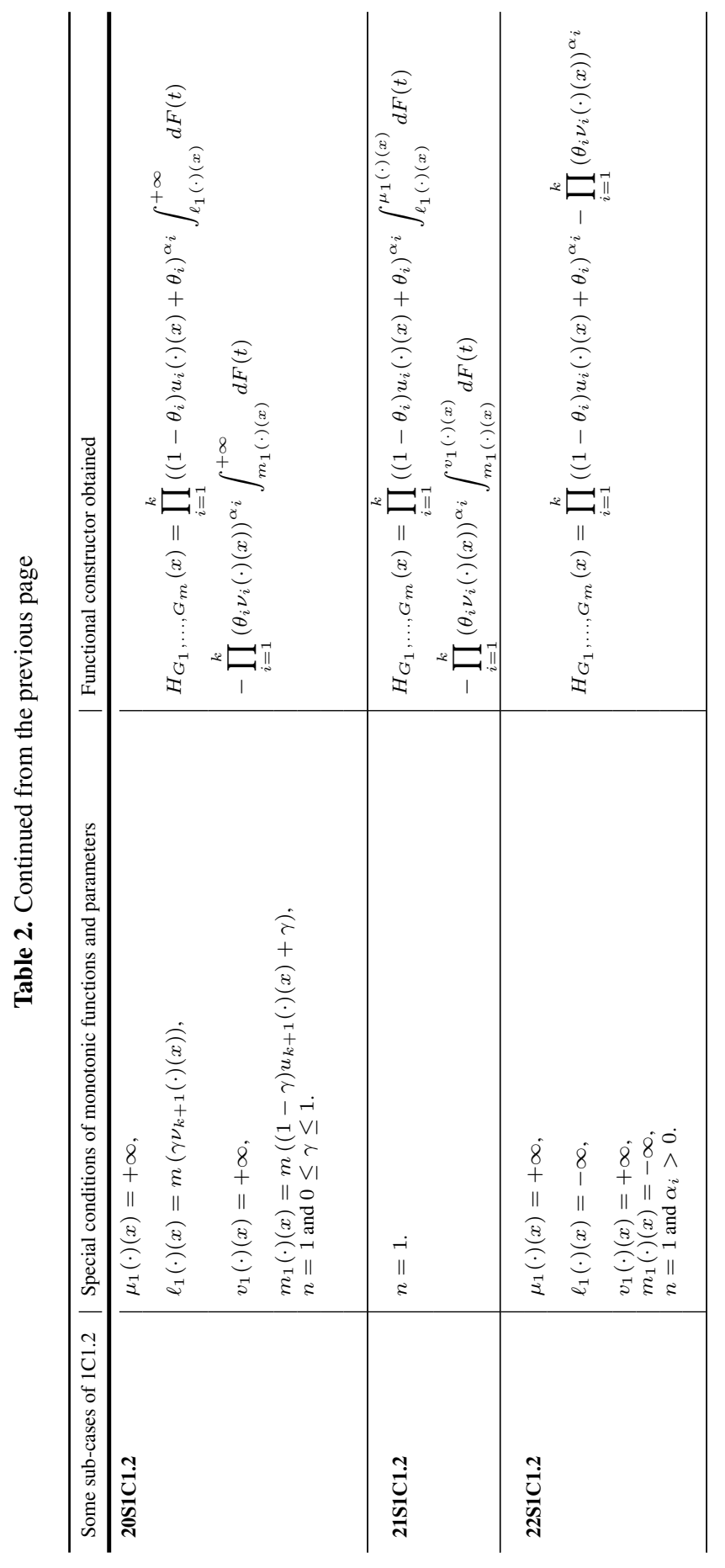


Corollary 1.3 shows an alternative method to obtain classes of probability distributions from Corollary 1.1. It shows what hypotheses $U, \vartheta, \mu_{j}, \ell_{j}, v_{j}$ and $m_{j}$ must satisfy so that the functions $\mho(x), W(x), \mathbb{U}_{j}(x), \mathbb{L}_{j}(x), \mathbb{M}_{j}(x)$ and $\mathbb{V}_{j}(x)$ satisfy the conditions of Corollary 1.1 and classes of probability distributions can be obtained.

1.3. Corollary $(\mathrm{C} 1.3)$. Complementary method to generate classes of probability distributions. Let $\varphi: \mathbb{R} \rightarrow \mathbb{R}, \mu_{j}:[0,1]^{m} \rightarrow \mathbb{R} \cup\{ \pm \infty\}, \ell_{j}:[0,1]^{m} \rightarrow \mathbb{R} \cup\{ \pm \infty\}, u:[0,1]^{m} \rightarrow \mathbb{R}$, $v_{j}:[0,1]^{m} \rightarrow \mathbb{R} \cup\{ \pm \infty\}, m_{j}:[0,1]^{m} \rightarrow \mathbb{R} \cup\{ \pm \infty\}$ and $\vartheta:[0,1]^{m} \rightarrow \mathbb{R}, \forall j=1,2,3, \ldots, \eta$, be monotonic and right continuous functions such that:

$[c d 1] \varphi$ is a cdf and $U$ and $\vartheta$ are non-negative;

$[c d 2] \mu_{j}, m_{j}$ and $U$ are non-decreasing and $\ell_{j}, v_{j}$ and $\vartheta$ are non-increasing, $\forall j=1,2,3, \ldots, \eta$, in all of its variables;

[cd3] If $U(1, \ldots, 1) \neq \vartheta(1, \ldots, 1)$, then $\vartheta(1, \ldots, 1)=0$ or $m_{j}(1, \ldots, 1)=v_{j}(1, \ldots, 1), \forall$ $j=1,2,3, \ldots, \eta$, and $u(1, \ldots, 1)=0$ or $\ell_{j}(1, \ldots, 1)=\mu_{j}(1, \ldots, 1), \forall j=1,2,3, \ldots, \eta$;

[cd4] If $U(1, \ldots, 1)=\vartheta(1, \ldots, 1) \neq 0$, then $\mu_{j}(1, \ldots, 1)=v_{j}(1, \ldots, 1), \forall j=1,2,3, \ldots, \eta$, and $m_{j}(1, \ldots, 1)=\ell_{j}(1, \ldots, 1), \forall j=1,2,3, \ldots, \eta$;

$[c d 5] \ell_{j}(0, \ldots, 0) \leq \mu_{j}(0, \ldots, 0)$ and if $\vartheta(1, \ldots, 1) \neq 0$, then $m_{j}(1, \ldots, 1) \leq v_{j}(1, \ldots$, $1), \forall j=1,2,3, \ldots, \eta$;

$[c d 6] v_{n}(0, \ldots, 0) \geq \sup \{x \in \mathbb{R}: F(x)<1\}$ and $m_{1}(0, \ldots, 0) \leq \inf \{x \in \mathbb{R}: F(x)>0\} ;$

$[c d 7] \vartheta(0, \ldots, 0)=1$

$[c d 8] U(0, \ldots, 0)=0$ or $\ell_{j}(0, \ldots, 0)=\mu_{j}(0, \ldots, 0), \forall j=1,2,3, \ldots, \eta-1$ and $\eta \geq 1$;

$[c d 9] v_{j}(0, \ldots, 0)=m_{j+1}(0, \ldots, 0), \forall j=1,2,3, \ldots, \eta-1$ and $\eta \geq 2$;

$[c d 10] \varphi$ is a cdf without points of discontinuity or the functions $\ell_{j}(\cdot)(x)$ and $v_{j}(\cdot)(x)$ are constant at the right of the vicinity of points whose image are points of discontinuity of $\varphi$, being also continuous in that points. Moreover, $\varphi$ does not have any point of discontinuity in the set $\left\{\ell_{j}(0, \ldots, 0)\right.$, $\mu_{j}(0, \ldots, 0), m_{j}(0, \ldots, 0), v_{j}(0, \ldots, 0), \ell_{j}(1, \ldots, 1), \mu_{j}(1, \ldots, 1), m_{j}(1, \ldots, 1), v_{j}(1, \ldots, 1)$, for some $j=1,2, \ldots, \eta\}$.

Then,

$$
H_{G_{1}, \ldots, G_{m}}(x)=1-\vartheta(\cdot)(x) \sum_{j=1}^{\eta} \int_{m_{j}(\cdot)(x)}^{v_{j}(\cdot)(x)} d \varphi(t)+\boldsymbol{u}(\cdot)(x) \sum_{j=1}^{\eta} \int_{\ell_{j}(\cdot)(x)}^{\mu_{j}(\cdot)(x)} d \varphi(t),
$$

is a functional generator of classes of probability distributions, where $(\cdot)(x)=\left(G_{1}, \ldots\right.$, $\left.G_{m}\right)(x)$.

Proof. In Corollary 1.1, set $\mho(x)=u(\cdot)(x), W(x)=\vartheta(\cdot)(x), \mathbb{U}_{j}(x)=\mu_{j}(\cdot)(x), \mathbb{L}_{j}(x)=$ $\ell_{j}(\cdot)(x), \mathbb{M}_{j}(x)=m_{j}(\cdot)(x)$ and $\mathbb{V}_{j}(x)=v_{j}(\cdot)(x)$ and observe that condition $[c d i]$ implies condition $[$ cci] of Corollary 1.1, for $i=1,2, \ldots, 10$.

Let us now consider a special case of Corollary 1.3 that is a functional constructor of classes of probability distributions that can be easily used. 
$1^{\text {st }}$ special case of Corollary 1.3 (1C1.3). Easy to use complementary method for the construction of classes of probability distributions.

Let $u_{i}:[0,1]^{m} \rightarrow[0,1]$ and $\nu_{i}:[0,1]^{m} \rightarrow[0,1]$ be monotonic and right continuous functions such that $u_{i}$ 's are non-decreasing and $\nu_{i}$ 's are non-increasing in each one of its variables, with $u_{i}(0, \ldots, 0)=0, u_{i}(1, \ldots, 1)=1, \nu_{i}(0, \ldots, 0)=1$ and $\nu_{i}(1, \ldots, 1)=0$, for all $i=1, \ldots, k$. If, in Corollary 1.3, $\vartheta(\cdot)(x)=\prod_{i=1}^{k}\left(\left(i-\theta_{i}\right) \nu_{i}(\cdot)(x)+\theta_{i}\right)^{\alpha_{i}}$ and $u(\cdot)(x)=$ $\prod_{i=1}^{k}\left(\theta_{i} u_{i}(\cdot)(x)\right)^{\alpha_{i}}$, with $\alpha_{i} \geq 0$ and $0 \leq \theta_{i} \leq 1$, then

$$
\begin{aligned}
H_{G_{1}, \ldots, G_{m}}(x) & =1-\prod_{i=1}^{k}\left(\left(1-\theta_{i}\right) \nu_{i}(\cdot)(x)+\theta_{i}\right)^{\alpha_{i}} \sum_{j=1}^{n} \int_{m_{j}(\cdot)(x)}^{v_{j}(\cdot)(x)} d \varphi(t) \\
& +\prod_{i=1}^{k}\left(\theta_{i} u_{i}(\cdot)(x)\right)^{\alpha_{i}} \sum_{j=1}^{n} \int_{\ell_{j}(\cdot)(x)}^{\mu_{j}(\cdot)(x)} d \varphi(t),
\end{aligned}
$$

is a functional generator of classes of probability distributions, where $(\cdot)(x)=\left(G_{1}, \ldots\right.$, $\left.G_{m}\right)(x)$.

Table 3 shows how to obtain some special cases of the function given by Equation (3.2), that may be more easily used to generate classes of distributions. It is important to emphasize that we can obtain the same constructors from 1S1C1.2 to 12S1C1.2 using 1C1.3, we omit the details here showing only how to obtain different constructors from those of Table 2. Consider the following functions in the expressions from 15S1C1.3 to 20S1C1.3: $\mu:[0,1] \rightarrow \mathbb{R} \cup\{ \pm \infty\}, \ell:[0,1] \rightarrow$ $\mathbb{R} \cup\{ \pm \infty\}, v:[0,1] \rightarrow \mathbb{R} \cup\{ \pm \infty\}, m:[0,1] \rightarrow \mathbb{R} \cup\{ \pm \infty\}$ such that $\mu$ and $m$ are non-decreasing and right continuous, and $v$ and $\ell$ are non-increasing and right continuous. 


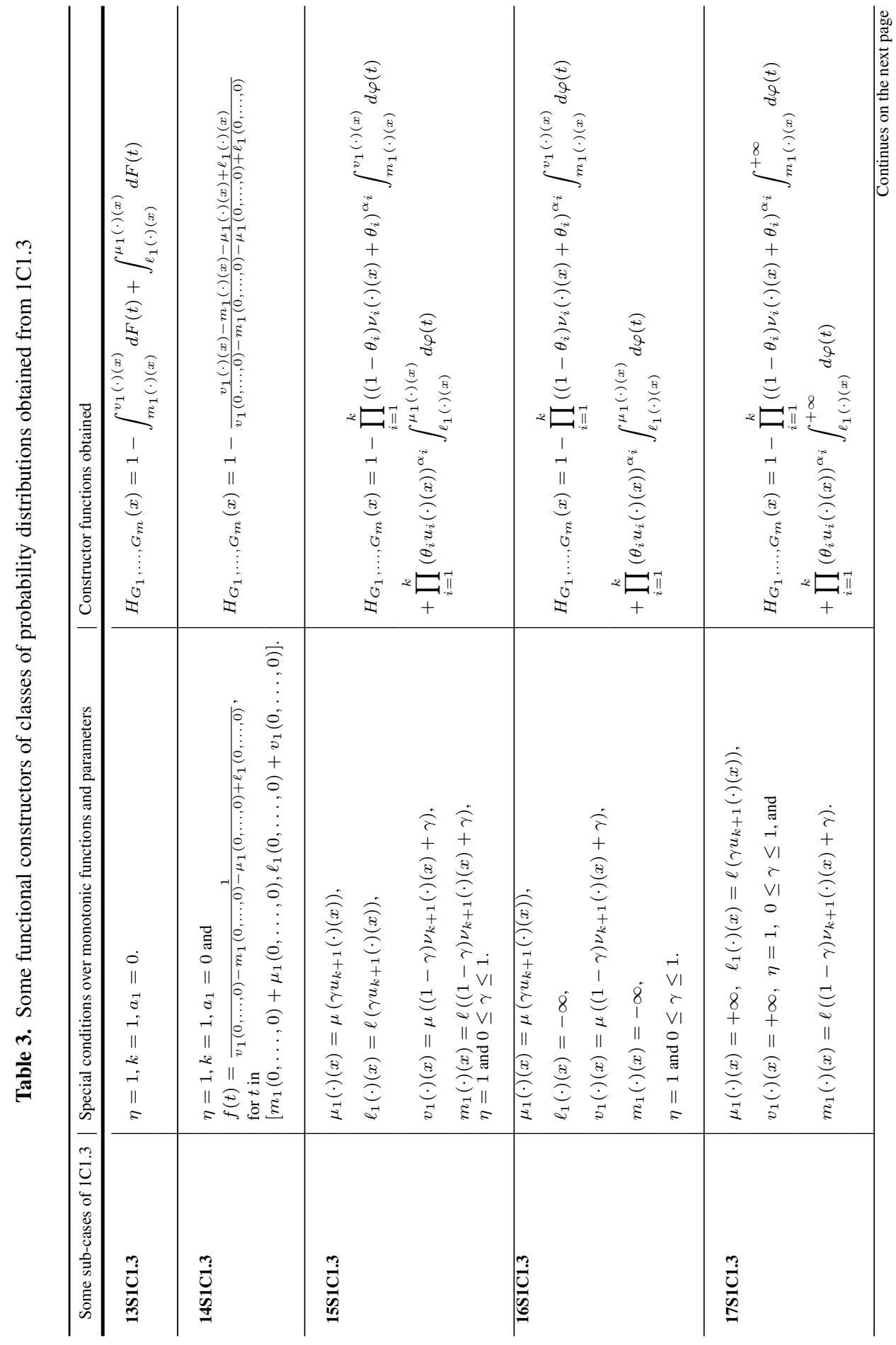




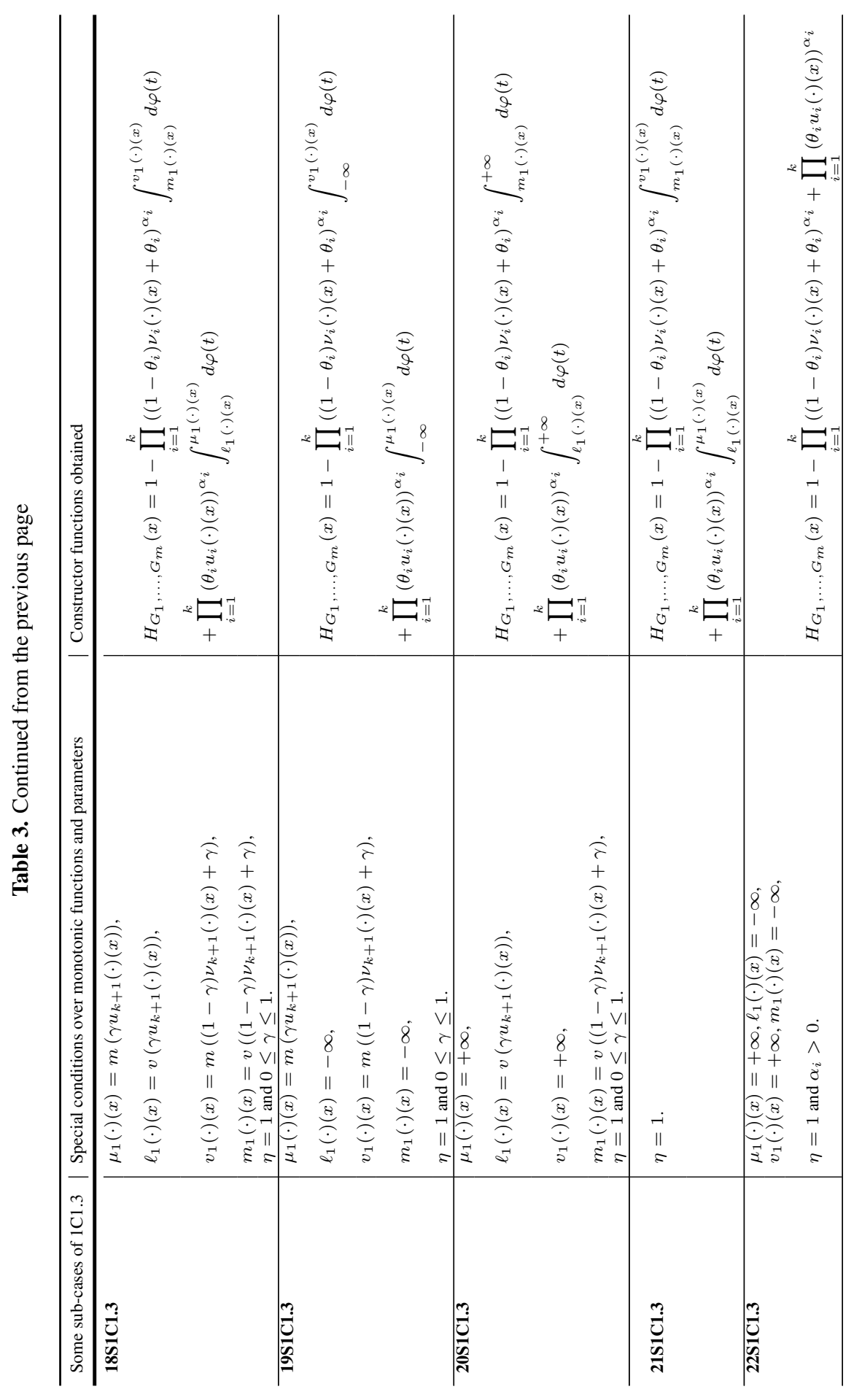


The following theorem shows that Theorem 1 and of its corollaries are equivalent. In other words, Theorem 1 and all of its corollaries generate the same probabilistic distributions.

2. Theorem (T2). Equivalence among Theorem 1 and its corollaries.

Theorem 1 and all of its corollaries generate exactly the same probabilistic distributions

Proof. To demonstrate Theorem 2 we show that $\mathrm{C} 1.1$ is a corollary of $\mathrm{T} 1$, that $\mathrm{C} 1.2$ is a corollary of $\mathrm{C} 1.1$, that $\mathrm{C} 1.3$ is a corollary of $\mathrm{C} 1.2$, and finally that $\mathrm{T} 1$ is a corollary of $\mathrm{C} 1.3$.

(1) C1.1 is a corollary of T1: it is obvious, as it has been already demonstrated.

(2) $\mathrm{C} 1.2$ is a corollary of C1.1: In Corollary $1.1, W(x)=1, \eta=1, \mathbb{V} 1(x)=1, \mathbb{M}_{1}(x)=$ $u(\cdot)(x) \sum_{j=1}^{n} \int_{\ell_{j}(\cdot)(x)}^{\mu_{j}(\cdot)(x)} d F(t)-\vartheta(\cdot)(x) \sum_{j=1}^{n} \int_{m_{j}(\cdot)(x)}^{v_{j}(\cdot)(x)} d F(t), \mho(x)=0, \forall x \in \mathbb{R}$ and $\varphi(t)$ the $c d f$ of the uniform $[0,1]$.

(3) $\mathrm{C} 1.3$ is a corollary of C1.2: In Corollary 1.2 , set $n=1, u(\cdot)(x)=1, \mu_{1}(\cdot)(x)=1$, $\ell_{1}(\cdot)(x)=0, \vartheta(\cdot)(x)=1, m_{1}(\cdot)(x)=0, v_{1}(\cdot)(x)=\vartheta(\cdot)(x) \sum_{j=1}^{\eta} \int_{m_{j}(\cdot)(x)}^{v_{j}(\cdot)(x)} d \varphi(t)-$ $u(\cdot)(x) \sum_{j=1}^{\eta} \int_{\ell_{j}(\cdot)(x)}^{\mu_{j}(\cdot)(x)} d \varphi(t), \forall x \in \mathbb{R}$ and $F(t)$ as the $c d f$ of the uniform $[0,1]$.

(4) $\mathrm{T} 1$ is a corollary of C1.3: In Corollary 1.3 , set $\eta=1, u(\cdot)(x)=1, \vartheta(\cdot)(x)=1, v_{1}(\cdot)(x)=1$, $m_{1}(\cdot)(x)=0, \ell_{1}(\cdot)(x)=0, \mu_{1}(\cdot)(x)=V(x) \sum_{j=1}^{n} \int_{L_{j}(x)}^{U_{j}(x)} d F(t)-\sqrt{ }(x) \sum_{j=1}^{n} \int_{M_{j}(x)}^{V_{j}(x)} d F(t)$ $\forall x \in \mathbb{R}$, and $\varphi(t) c d f$ of the uniform $[0,1]$.

From (1) to (4), we conclude Theorem 2.

Several classes of probability distributions existing in the literature can be obtained as special cases of the functional constructors of classes of probability distributions proposed here. Table 4, in the Appendix A, shows how to obtain such classes using some corollaries of Theorem 1.

\section{Support of the classes of probability distributions}

In this section, we provide an analysis about the support and nature of the probability distributions generated through the methods described in Corollaries 1.2 and 1.3. These results are important to gain a deeper understanding about the proposed method, especially considering the fact that there is little work on this theme in the literature.

In order to state the results, we remind the reader that, by the definition, the support of a cumulative distribution function $F$ is given by $S_{F}=\{x \in \mathbb{R}:(x)-F(x-\varepsilon)>0, \forall \varepsilon>0\}$. Theorem 3 shows that the support of the generated distribution is contained in the union of the supports of the baseline distributions $G_{i}$ 's.

3. Theorem (T3). General theorem of the supports.

Let $H_{G_{1}, \ldots, G_{m}}(x)$ be a cumulative distribution function generated from Corollary 1.2 (respectively, 1.3). Then,

$$
S_{H_{G_{1}, \ldots, G_{m}}} \subset \cup_{j=1}^{m} S_{G_{j}} .
$$

Proof. Consider that $H_{G_{1}, \ldots, G_{m}}(x)$ has the functional form of Corollary 1.2:

$$
H_{G_{1}, \ldots, G_{m}}(x)=U(\cdot)(x) \sum_{j=1}^{n} \int_{\ell_{j}(\cdot)(x)}^{\mu_{j}(\cdot)(x)} d F(t)-\vartheta(\cdot)(x) \sum_{j=1}^{n} \int_{m_{j}(\cdot)(x)}^{v_{j}(\cdot)(x)} d F(t) .
$$


Thus, it follows that

$$
\begin{aligned}
H_{G_{1}, \ldots, G_{m}}(x)-H_{G_{1}, \ldots, G_{m}}(x-\varepsilon) & =u(\cdot)(x) \sum_{j=1}^{n} \int_{\ell_{j}(\cdot)(x)}^{\mu_{j}(\cdot)(x)} d F(t) \\
& -\vartheta(\cdot)(x) \sum_{j=1}^{n} \int_{m_{j}(\cdot)(x)}^{v_{j}(\cdot)(x)} d F(t) \\
& -U(\cdot)(x-\varepsilon) \sum_{j=1}^{n} \int_{\ell_{j}(\cdot)(x-\varepsilon)}^{\mu_{j}(\cdot)(x-\varepsilon)} d F(t) \\
& +\vartheta(\cdot)(x-\varepsilon) \sum_{j=1}^{n} \int_{m_{j}(\cdot)(x-\varepsilon)}^{v_{j}(\cdot)(x-\varepsilon)} d F(t) .
\end{aligned}
$$

Suppose that $x \notin \cup_{j=1}^{m} S_{G_{j}}$. Then, there exists $\varepsilon>0$ such that $G_{j}(x)-G_{j}(x-\varepsilon)=0$, for all $j=1,2, \ldots, m$. Let us show that $H_{G_{1}, \ldots, G_{m}}(x)-H_{G_{1}, \ldots, G_{m}}(x-\varepsilon)=0$.

First, note that

$$
\begin{aligned}
H_{G_{1}, \ldots, G_{m}}(x) & -H_{G_{1}, \ldots, G_{m}}(x-\varepsilon)=(U(\cdot)(x)-U(\cdot)(x-\varepsilon)) \sum_{j=1}^{n} \int_{\ell_{j}(\cdot)(x)}^{\mu_{j}(\cdot)(x)} d F(t) \\
& -(\vartheta(\cdot)(x)-\vartheta(\cdot)(x-\varepsilon)) \int_{m_{j}(\cdot)(x)}^{v_{j}(\cdot)(x)} d F(t) \\
& +U(\cdot)(x-\varepsilon) \sum_{j=1}^{n}\left\{\int_{\mu_{j}(\cdot)(x-\varepsilon)}^{\mu_{j}(\cdot)(x)} d F(t)-\int_{\ell_{j}(\cdot)(x-\varepsilon)}^{\ell_{j}(\cdot)(x)} d F(t)\right\} \\
& -\vartheta(\cdot)(x-\varepsilon) \sum_{j=1}^{n}\left\{\int_{v_{j}(\cdot)(x-\varepsilon)}^{v_{j}(\cdot)(x)} d F(t)-\int_{m_{j}(\cdot)(x-\varepsilon)}^{m_{j}(\cdot)(x)} d F(t)\right\} .
\end{aligned}
$$

Since $U(\cdot)(x)=U(\cdot)(x-\varepsilon), \vartheta(\cdot)(x)=\vartheta(\cdot)(x-\varepsilon), \mu_{j}(\cdot)(x)=\mu_{j}(\cdot)(x-\varepsilon), \ell_{j}(\cdot)(x)=$ $\ell_{j}(\cdot)(x-\varepsilon), m_{j}(\cdot)(x)=m_{j}(\cdot)(x-\varepsilon), v_{j}(\cdot)(x)=v_{j}(\cdot)(x-\varepsilon)$, it follows that

$$
H_{G_{1}, \ldots, G_{m}}(x)-H_{G_{1}, \ldots, G_{m}}(x-\varepsilon)=0 .
$$

Thus, we have $x \notin S_{H_{G_{1}, \ldots, G_{m}}}$. Therefore, $S_{H_{G_{1}, \ldots, G_{m}}} \subset \cup_{j=1}^{m} S_{G_{j}}$. A similar argument works for the case where $H_{G_{1}, \ldots, G_{m}}(x)$ has the functional form of Corollary 1.3.

Corollary 3.1 shows a special case where the distribution $H_{G_{1}, \ldots, G_{m}}(x)$ is discrete.

3.1. Corollary (C3.1). Discrete baselines generate discrete distributions.

If all $G_{j}$ 's are discrete in Corollary 1.2 (respectively, 1.3), then $H_{G_{1}, \ldots, G_{m}}(x)$ is discrete.

Proof. Being all $G_{j}$ 's discrete, then $\cup_{j=1}^{m} S_{G_{j}}$ has a countable number of values. Since, by Theorem 3, $S_{H_{G_{1}}, \ldots, G_{m}} \subset \cup_{j=1}^{m} S_{G_{j}}$, then $S_{H_{G_{1}}, \ldots, G_{m}}$ has a countable number of values and, for this reason, $H_{G_{1}, \ldots, G_{m}}(x)$ is acdf of a discrete random variable.

Theorem 4 shows conditions when $S_{H_{G_{1}, \ldots, G_{m}}} \subset \cup_{j=1}^{m} S_{G_{j}}$.

4. Theorem (T4). The support of distribution is a union of the supports of the baselines. Assume, in Corollary 1.2, (respectively, 1.3) that:

[f1] $S_{F}$ is a convex set;

$[f 2] \mu_{n}(1, \ldots, 1)=\sup \{x \in \mathbb{R}: \varphi(x)<1\}, \ell_{1}(1, \ldots, 1)=\inf \{x \in \mathbb{R}: \varphi(x)<1\}, U(\cdot)(x)>$ $0, \forall x \in \mathbb{R}$, and that $\mu_{j}(\cdot)(x)$ or $\ell_{j}(\cdot)(x)$, for some $j=1,2, \ldots, n$, are strictly monotonic or $v_{n}(0, \ldots, 0)=\sup \{x \in \mathbb{R}: \varphi(x)<1\}, m_{1}(0, \ldots, 0)=\inf \{x \in \mathbb{R}: \varphi(x)>0\}, \vartheta(\cdot)(x)>0$, $\forall x \in \mathbb{R}$, and that $v_{j}(\cdot)(x)$ or $m_{j}(\cdot)(x)$, for some $j=1,2, \ldots, n$, are strictly monotonic. 
Then,

$$
S_{H_{G_{1}, \ldots, G_{m}}} \subset \cup_{j=1}^{m} S_{G_{j}} .
$$

Proof. As a consequence of Theorem 3, we only need to show that

$$
S_{H_{G_{1}, \ldots, G_{m}}} \subset \cup_{j=1}^{m} S_{G_{j}} .
$$

Consider that $H_{G_{1}, \ldots, G_{m}}(x)$ has the functional form of Corollary 1.2:

$H_{G_{1}, \ldots, G_{m}}(x)=U(\cdot)(x) \sum_{j=1}^{n} \int_{\ell_{j}(\cdot)(x)}^{\mu_{j}(\cdot)(x)} d F(t)-\vartheta(\cdot)(x) \sum_{j=1}^{n} \int_{m_{j}(\cdot)(x)}^{v_{j}(\cdot)(x)} d F(t)$.

Thus, using an argument identical to that of the proof of Theorem 3 , we have

$$
\begin{aligned}
H_{G_{1}, \ldots, G_{m}}(x) & -H_{G_{1}, \ldots, G_{m}}(x-\varepsilon)=(u(\cdot)(x)-u(\cdot)(x-\varepsilon)) \sum_{j=1}^{n} \int_{\ell_{j}(\cdot)(x)}^{\mu_{j}(\cdot)(x)} d F(t) \\
& -(\vartheta(\cdot)(x)-\vartheta(\cdot)(x-\varepsilon)) \int_{m_{j}(\cdot)(x)}^{v_{j}(\cdot)(x)} d F(t) \\
& +u(\cdot)(x-\varepsilon) \sum_{j=1}^{n}\left\{\int_{\mu_{j}(\cdot)(x-\varepsilon)}^{\mu_{j}(\cdot)(x)} d F(t)-\int_{\ell_{j}(\cdot)(x-\varepsilon)}^{\ell_{j}(\cdot)(x)} d F(t)\right\} \\
& -\vartheta(\cdot)(x-\varepsilon) \sum_{j=1}^{n}\left\{\int_{v_{j}(\cdot)(x-\varepsilon)}^{v_{j}(\cdot)(x)} d F(t)-\int_{m_{j}(\cdot)(x-\varepsilon)}^{m_{j}(\cdot)(x)} d F(t)\right\} .
\end{aligned}
$$

Suppose that $x \in \cup_{j=1}^{m} S_{G_{j}}$. Then, there exists $\varepsilon>0$ such that $G_{j}(x)-G_{j}(x-\varepsilon)>0$ for some $j=1,2, \ldots, m$. Conditions $[f 1]$ and $[f 2]$ imply that at least one of the integrals of the form $\int_{h(\cdot)(x-\varepsilon)}^{h(\cdot)(x)} d F(t)$ is different from zero for $h=\mu_{j}, h=\ell_{j}, h=v_{j}$ or $h=m_{j}$, for some $j=1,2, \ldots, n$. This fact together with the fact that $U$ or $\vartheta$ are strictly monotonic imply that:

$H_{G_{1}, \ldots, G_{m}}(x)-H_{G_{1}, \ldots, G_{m}}(x-\varepsilon)>0$. Thus, $x \in S_{H_{G_{1}, \ldots, G_{m}}}$, as desired. A similar argument works for the case where $H_{G_{1}, \ldots, G_{m}}(x)$ has the functional form of Corollary 1.3.

Theorem 5 shows some conditions that guarantee that $H_{G_{1}, \ldots, G_{m}}(x)$ is a continuous $c d f$.

5. Theorem (T5). Generating continuous cumulative distribution functions.

Suppose that $F(x), G_{1}, \ldots, G_{m}$ are continuous $c d f^{\prime} s$ and that $\mu_{j}, \ell_{j}, U, v_{j}, m_{j}$ and $\vartheta$ are continuous functions in Corollary 1.2 (respectively, 1.3). Then, $H_{G_{1}, \ldots, G_{m}}(x)$ is a continuous cdf.

Proof. Consider that $H_{G_{1}, \ldots, G_{m}}(x)$ has the functional form of Corollary 1.2

$$
H_{G_{1}, \ldots, G_{m}}(x)=U(\cdot)(x) \sum_{j=1}^{n} \int_{\ell_{j}(\cdot)(x)}^{\mu_{j}(\cdot)(x)} d F(t)-\vartheta(\cdot)(x) \sum_{j=1}^{n} \int_{m_{j}(\cdot)(x)}^{v_{j}(\cdot)(x)} d F(t) .
$$

Thus, it follows that

$$
\begin{aligned}
H_{G_{1}, \ldots, G_{m}}(x)-H_{G_{1}, \ldots, G_{m}}\left(x^{-}\right) & =u(\cdot)(x) \sum_{j=1}^{n} \int_{\ell_{j}(\cdot)(x)}^{\mu_{j}(\cdot)(x)} d F(t) \\
& -\vartheta(\cdot)(x) \sum_{j=1}^{n} \int_{m_{j}(\cdot)(x)}^{v_{j}(\cdot)(x)} d F(t) \\
& -u(\cdot)\left(x^{-}\right) \sum_{j=1}^{n} \int_{\ell_{j}(\cdot)\left(x^{-}\right)}^{\mu_{j}(\cdot)\left(x^{-}\right)} d F(t) \\
& +\vartheta(\cdot)(x) \sum_{j=1}^{n} \int_{m_{j}(\cdot)\left(x^{-}\right)}^{v_{j}(\cdot)\left(x^{-}\right)} d F(t)
\end{aligned}
$$


Using a similar approach as that used in the proof of Theorem 3, we obtain:

$$
\begin{aligned}
H_{G_{1}, \ldots, G_{m}}(x) & -H_{G_{1}, \ldots, G_{m}}\left(x^{-}\right)=\left(u(\cdot)(x)-u(\cdot)\left(x^{-}\right)\right) \sum_{j=1}^{n} \int_{\ell_{j}(\cdot)(x)}^{\mu_{j}(\cdot)(x)} d F(t) \\
& -\left(\vartheta(\cdot)(x)-\vartheta(\cdot)\left(x^{-}\right)\right) \sum_{j=1}^{n} \int_{m_{j}(\cdot)(x)}^{v_{j}(\cdot)(x)} d F(t) \\
& +U(\cdot)\left(x^{-}\right) \sum_{j=1}^{n}\left\{\int_{\mu_{j}(\cdot)\left(x^{-}\right)}^{\mu_{j}(\cdot)(x)} d F(t)-\int_{\ell_{j}(\cdot)\left(x^{-}\right)}^{\ell_{j}(\cdot)(x)} d F(t)\right\} \\
& -\vartheta(\cdot)\left(x^{-}\right)\left\{\sum_{j=1}^{n} \int_{v_{j}(\cdot)\left(x^{-}\right)}^{v_{j}(\cdot)(x)} d F(t)-\int_{m_{j}(\cdot)\left(x^{-}\right)}^{m_{j}(\cdot)(x)} d F(t)\right\} .
\end{aligned}
$$

Since all functions included in the previous expression are continuous, we have:

$$
H_{G_{1}, \ldots, G_{m}}(x)-H_{G_{1}, \ldots, G_{m}}\left(x^{-}\right)=0 .
$$

Therefore, we shall conclude that $H_{G_{1}, \ldots, G_{m}}(x)$ is a continuous function. A similar argument works for the case where $H_{G_{1}, \ldots, G_{m}}(x)$ has the functional form of Corollary 1.3.

Theorem 6 shows conditions where distribution $H_{G_{1}, \ldots, G_{m}}(x)$ will be a continuous $c d f$ of random variables.

6. Theorem (T6). Generating cumulative distribution functions of continuous random variables. Suppose that $F(x), G_{1}, \ldots, G_{m}$ are cdf's of continuous random variables and that $\mu_{j}, \ell_{j}, u, v_{j}$, $m_{j}$ and $\vartheta$ are continuous and differentiable functions in Corollary 1.2 (respectively, 1.3). Then, $H_{G_{1}, \ldots, G_{m}}(x)$ is a cdf of a continuous random variable.

Proof. Consider that $H_{G_{1}, \ldots, G_{m}}(x)$ has the functional form of Corollary 1.2

$H_{G_{1}, \ldots, G_{m}}(x)=U(\cdot)(x) \sum_{j=1}^{n} \int_{\ell_{j}(\cdot)(x)}^{\mu_{j}(\cdot)(x)} d F(t)-\vartheta(\cdot)(x) \sum_{j=1}^{n} \int_{m_{j}(\cdot)(x)}^{v_{j}(\cdot)(x)} d F(t)$.

Since $F(x), G_{1}, \ldots, G_{m}$ are $c d f^{\prime} s$ of continuous random variables and $\mu_{j}, \ell_{j}, u, v_{j}, m_{j}$ and $\vartheta$ are continuous and differentiable functions, then $H_{G_{1}, \ldots, G_{m}}(x)$ is a $c d f$ of a continuous random variable with density given by:

$$
\begin{aligned}
H_{G_{1}, \ldots, G_{m}}(x) & =\left(\sum_{z=1}^{m} \frac{\partial u(\cdot)(x)}{\partial G_{z}} g_{z}(x)\right)\left(\sum_{j=1}^{n} \int_{\ell_{j}(\cdot)(x)}^{\mu_{j}(\cdot)(x)} d F(t)\right) \\
& +u(\cdot)(x) \sum_{j=1}^{n}\left\{F \left(\mu_{j}(\cdot)(x) \sum_{z=1}^{m} \frac{\partial \mu_{j}(\cdot)(x)}{\partial G_{z}} g_{z}(x)\right.\right. \\
& \left.-F\left(\ell_{j}(\cdot)(x)\right) \sum_{z=1}^{m} \frac{\partial \ell_{j}(\cdot)(x)}{\partial G_{z}} g_{z}(x)\right\} \\
& -\left(\sum_{z=1}^{m} \frac{\partial \vartheta(\cdot)(x)}{\partial G_{z}} g_{z}(x)\right)\left(\sum_{j=1}^{n} \int_{m_{j}(\cdot)(x)}^{v_{j}(\cdot)(x)} d F(t)\right) \\
& -\vartheta(\cdot)(x) \sum_{j=1}^{n}\left\{F\left(v_{j}(\cdot)(x)\right) \sum_{z=1}^{m} \frac{\partial v_{j}(\cdot)(x)}{\partial G_{z}} g_{z}(x)\right. \\
& \left.-F\left(m_{j}(\cdot)(x)\right) \sum_{z=1}^{m} \frac{\partial m_{j}(\cdot)(x)}{\partial G_{z}} g_{z}(x)\right\}
\end{aligned}
$$


where $(\cdot)(x)=\left(G_{1}, \ldots, G_{m}\right)(x)$. A similar argument works for the case where $H_{G_{1}, \ldots, G_{m}}(x)$ has the functional form of Corollary 1.3 .

The next theorem shows an alternative way of generating discrete distributions.

7. Theorem (T7). Integrals with respect to discrete distributions generate discrete distributions. Suppose that the probability distribution $F(x)$ is discrete and that $u(\cdot)(x)=\vartheta(\cdot)(x)=1$, in Corollary 1.2 (respectively, 1.3). Then, $H_{G_{1}, \ldots, G_{m}}(x)$ is discrete independent from the monotonic functions that are used as limits of the integration.

Proof. We can write the following

$$
\begin{aligned}
H_{G_{1}, \ldots, G_{m}}(x) & =\sum_{j=1}^{n} \int_{\ell_{j}(\cdot)(x)}^{\mu_{j}(\cdot)(x)} d F(t)-\sum_{j=1}^{n} \int_{m_{j}(\cdot)(x)}^{v_{j}(\cdot)(x)} d F(t) \\
& =\sum_{j=1}^{n}\left[F\left(\mu_{j}(\cdot)(x)\right)-F\left(\ell_{j}(\cdot)(x)\right)\right] \\
& -\sum_{j=1}^{n}\left[F\left(v_{j}(\cdot)(x)\right)-F\left(m_{j}(\cdot)(x)\right)\right] .
\end{aligned}
$$

Since $F(x)$ is a $c d f$ of a discrete random variable, then $F(x)$ assumes a countable number of different values. Thus, as $H_{G_{1}, \ldots, G_{m}}(x)$ is given by the sum of differences of $F(x)$ evaluated in at most $4 n$ distinct points, it also can only assume a countable number of different values and, therefore, it is a $c d f$ of a discrete random variable.

\section{Conclusions}

The method to generate distributions and classes of probability distributions that we presented in this paper combines several methods to generate classes of distribution that have already been described in the literature. By this unification, we could draw conclusions on the supports of generated classes. Using the proposed method, we can generate any probability distribution in different ways. The only necessity is to modify the monotonic functions involved in the method.

As a further step, we aim to explore several classes of distribution which may be generated using this method, developing their properties and applying them to model several datasets. In a parallel work, we are proposing a method for generating multivariate distributions.

It is important to stress that a model can better describe a phenomenon by increasing its number of parameters, providing higher flexibility. On the other hand, we should not forget that increasing the number of parameters may cause identifiability and computational problems in the estimation of the parameters. Moreover, a large number of parameters increase the chance of overfitting, which is a problem particularly in forecasting and prediction studies. Thus, the best approach is to choose a method that best describes the analyzed phenomenon or experiment with the lowest possible number of parameters.

\section{Appendix A. how to obtain generalizations of already existing class models}

In this appendix we will show some applications to obtain some very special examples of functions generating classes of probabilistic distributions by finding probability distribution classes that have already been described in literature.

Table 4 shows how to obtain classes of probability distributions already existing in the literature by the use of some corollaries of Theorem 1 . 


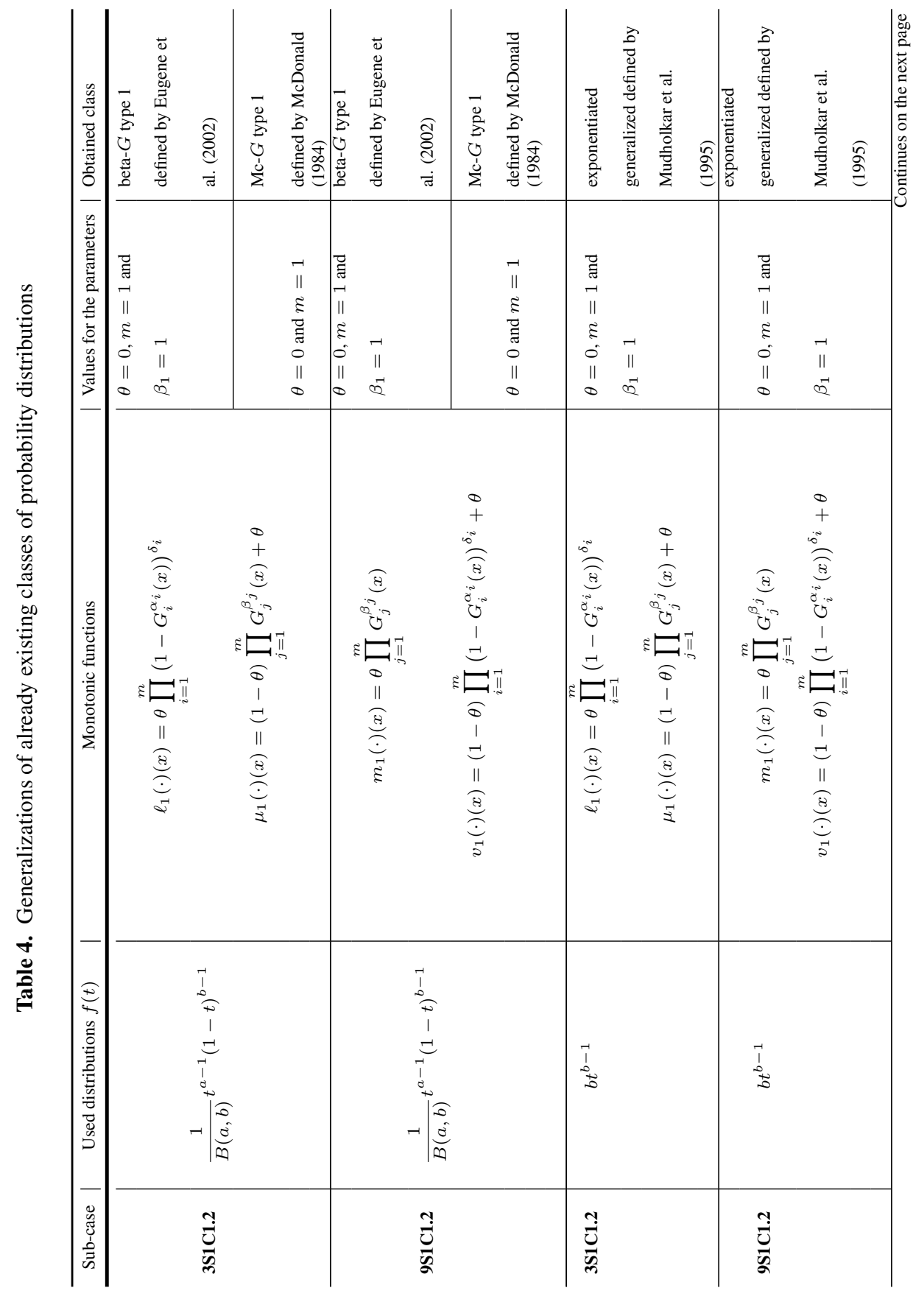




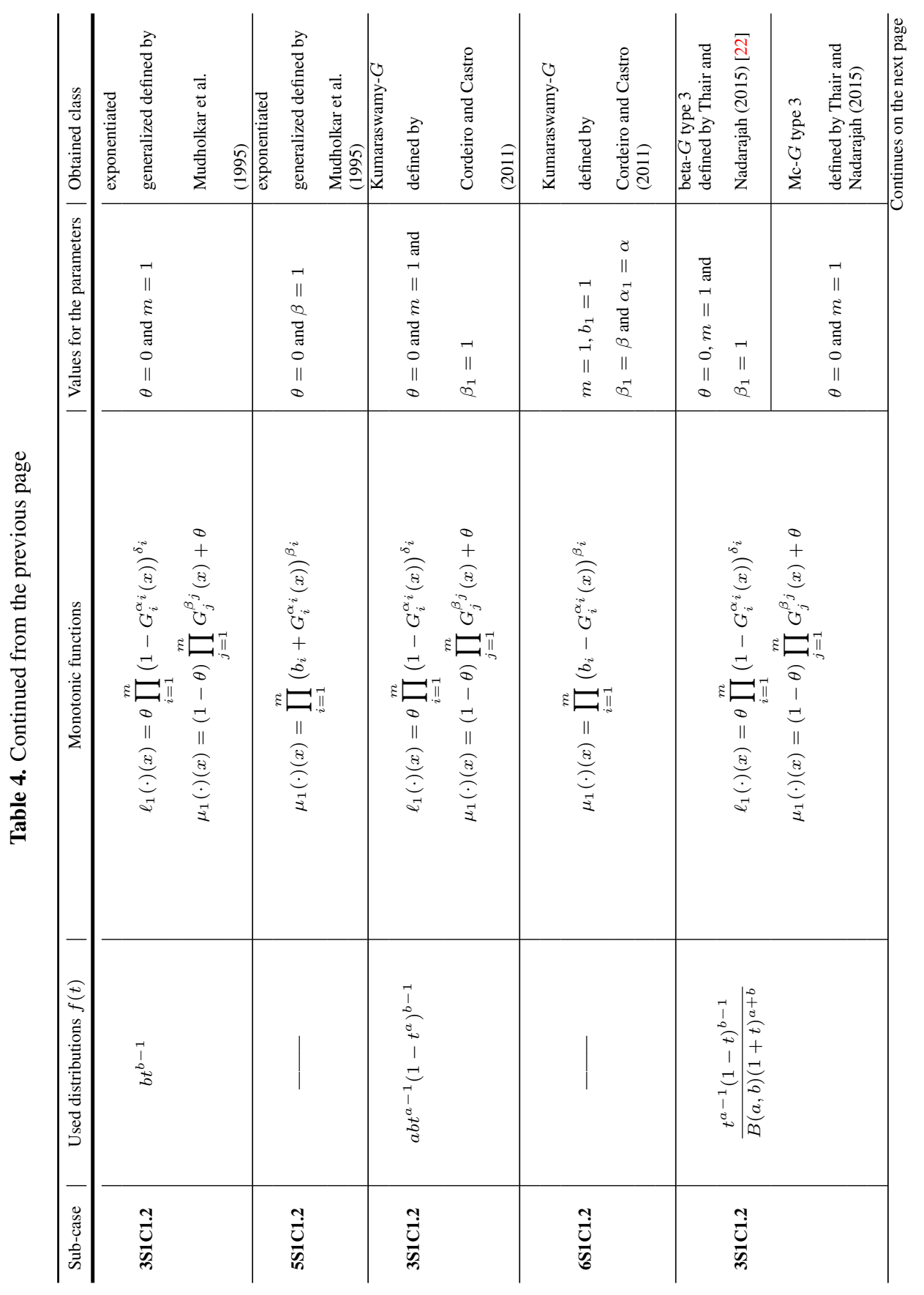




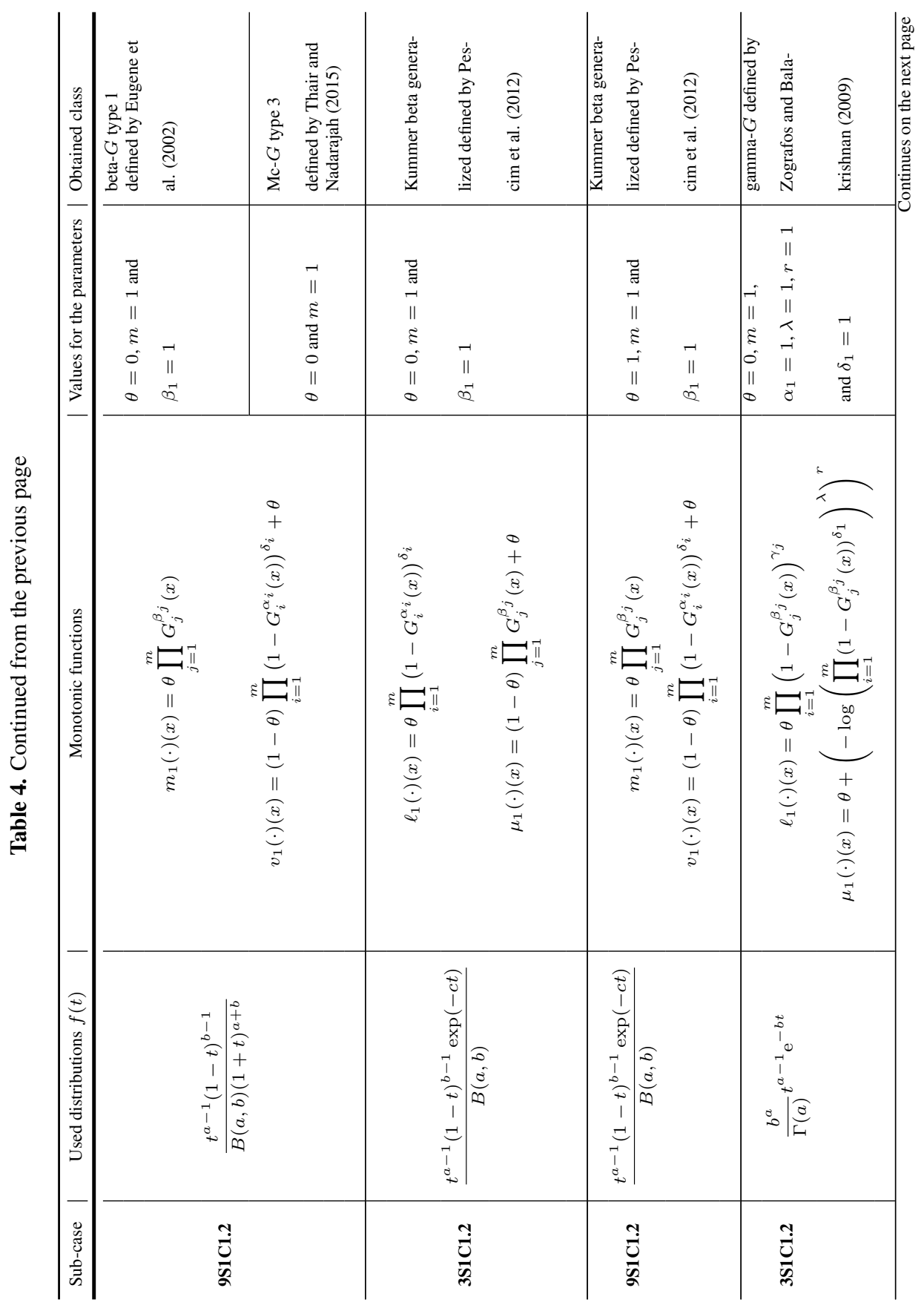




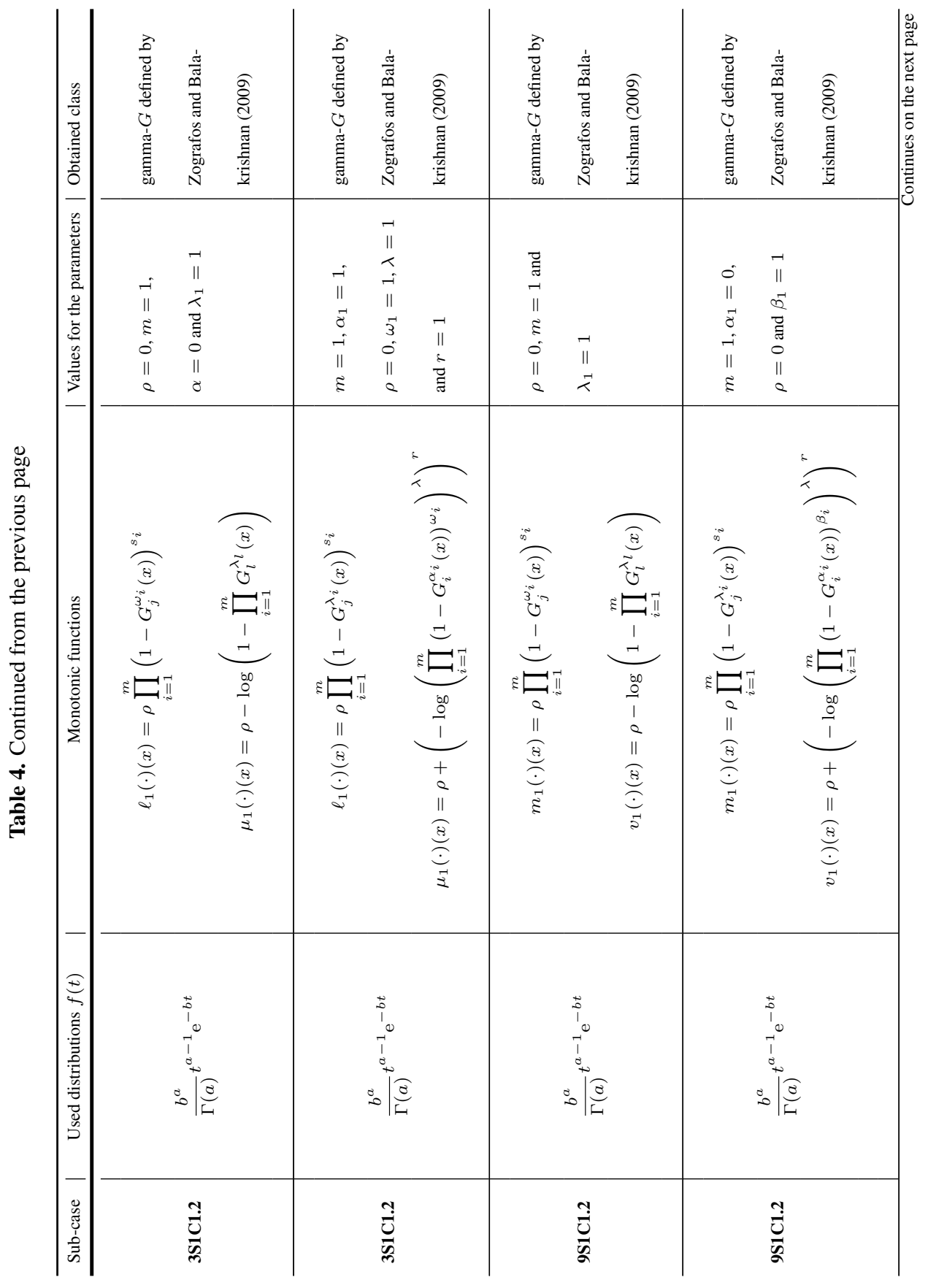




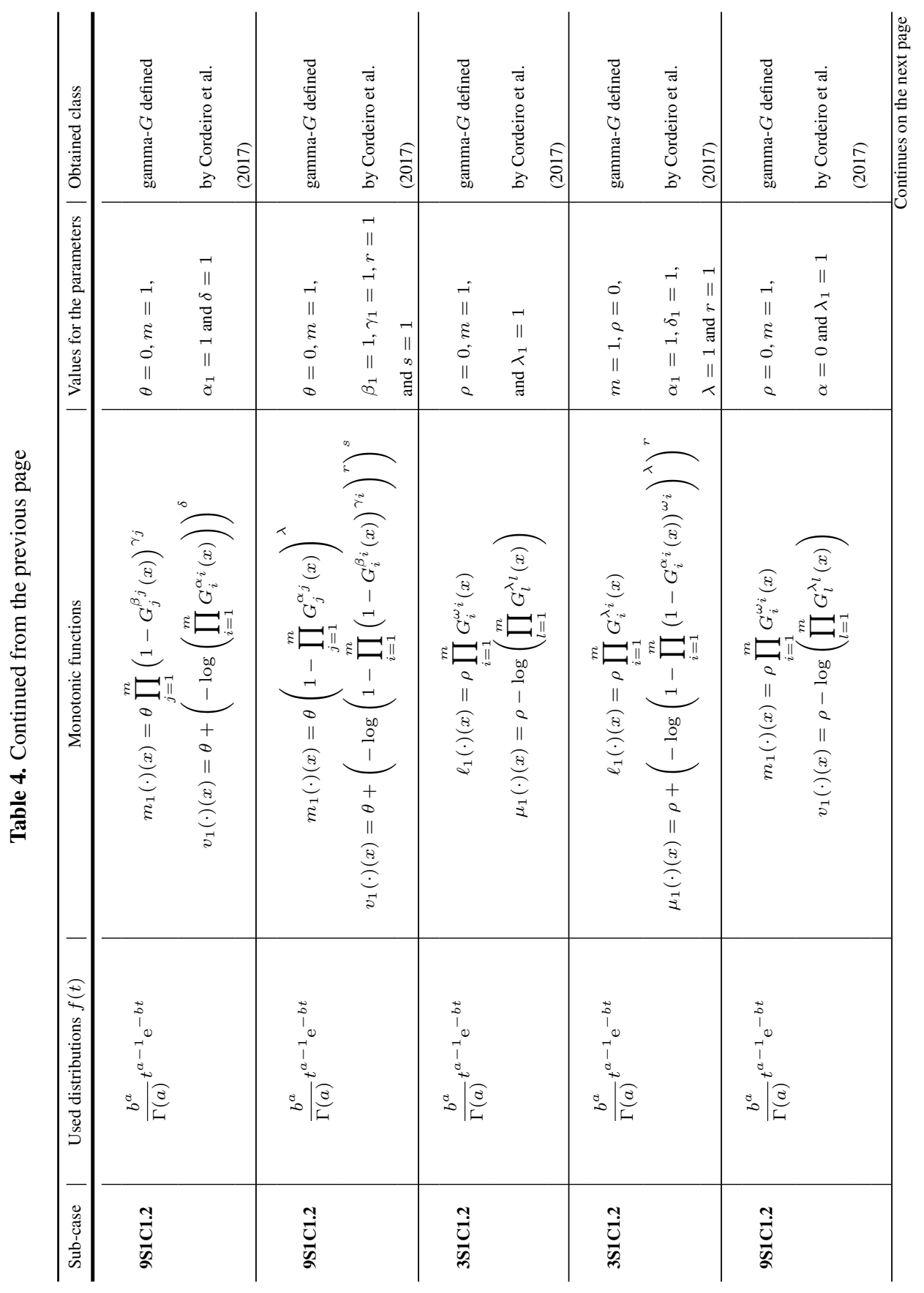




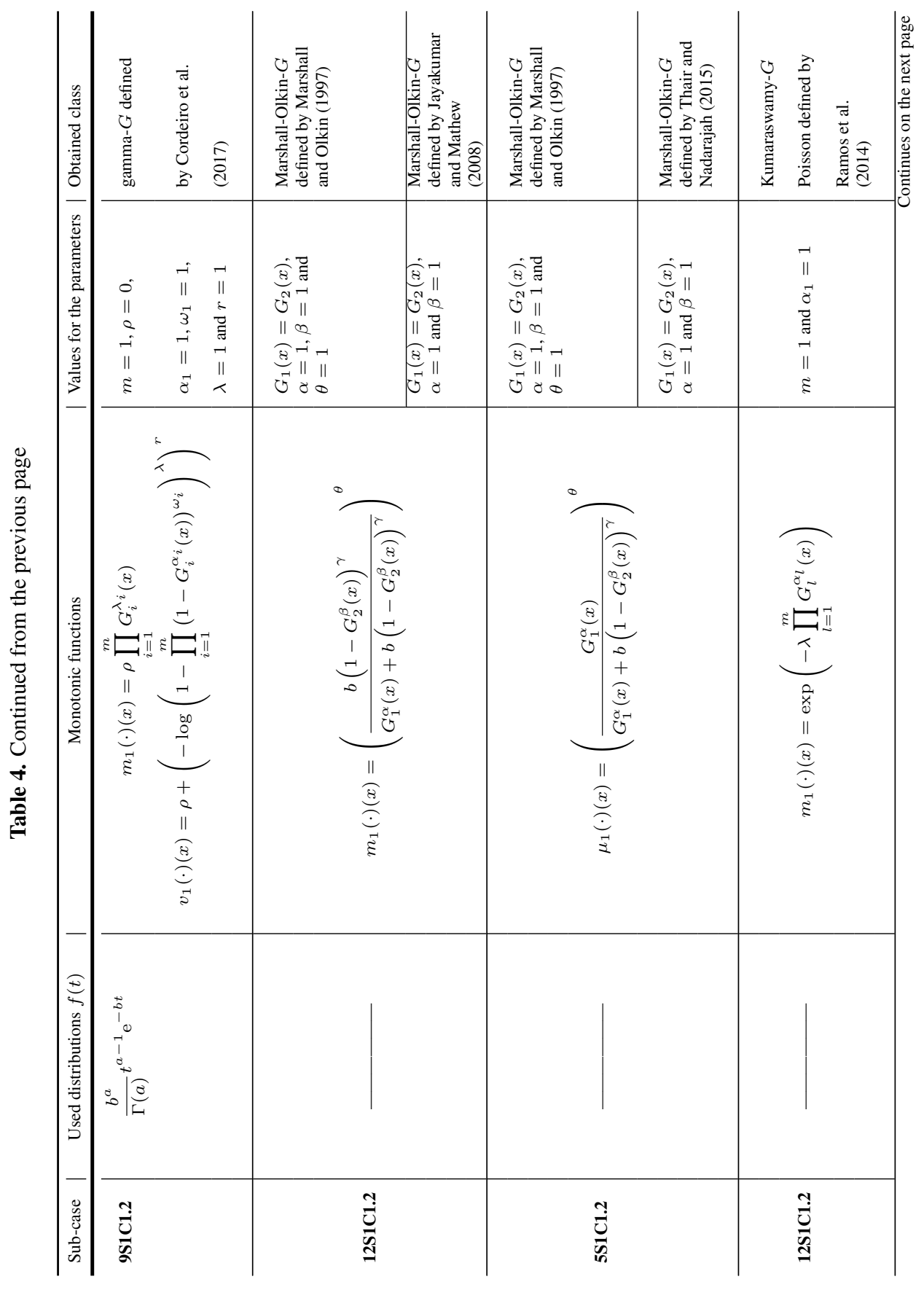




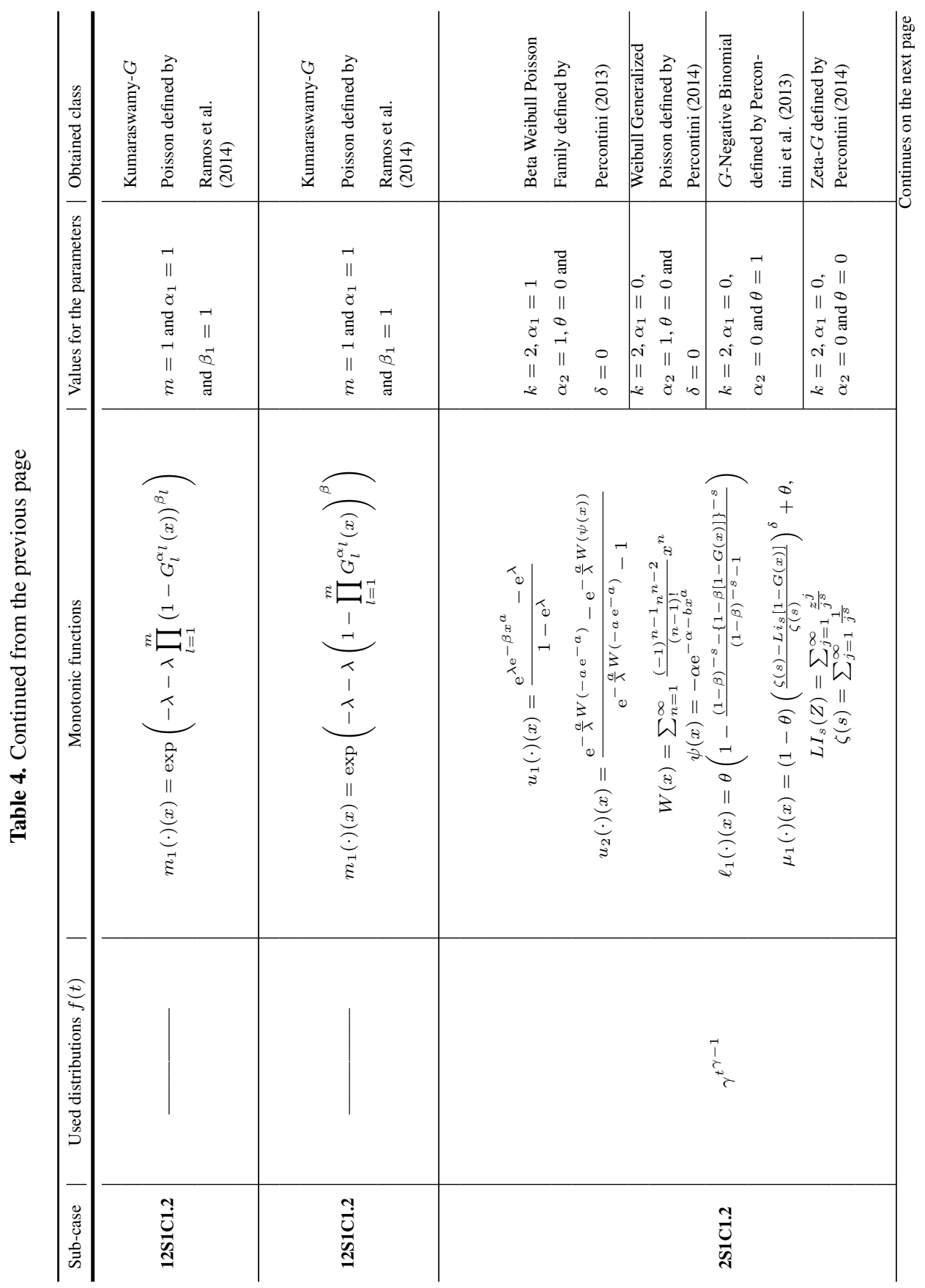




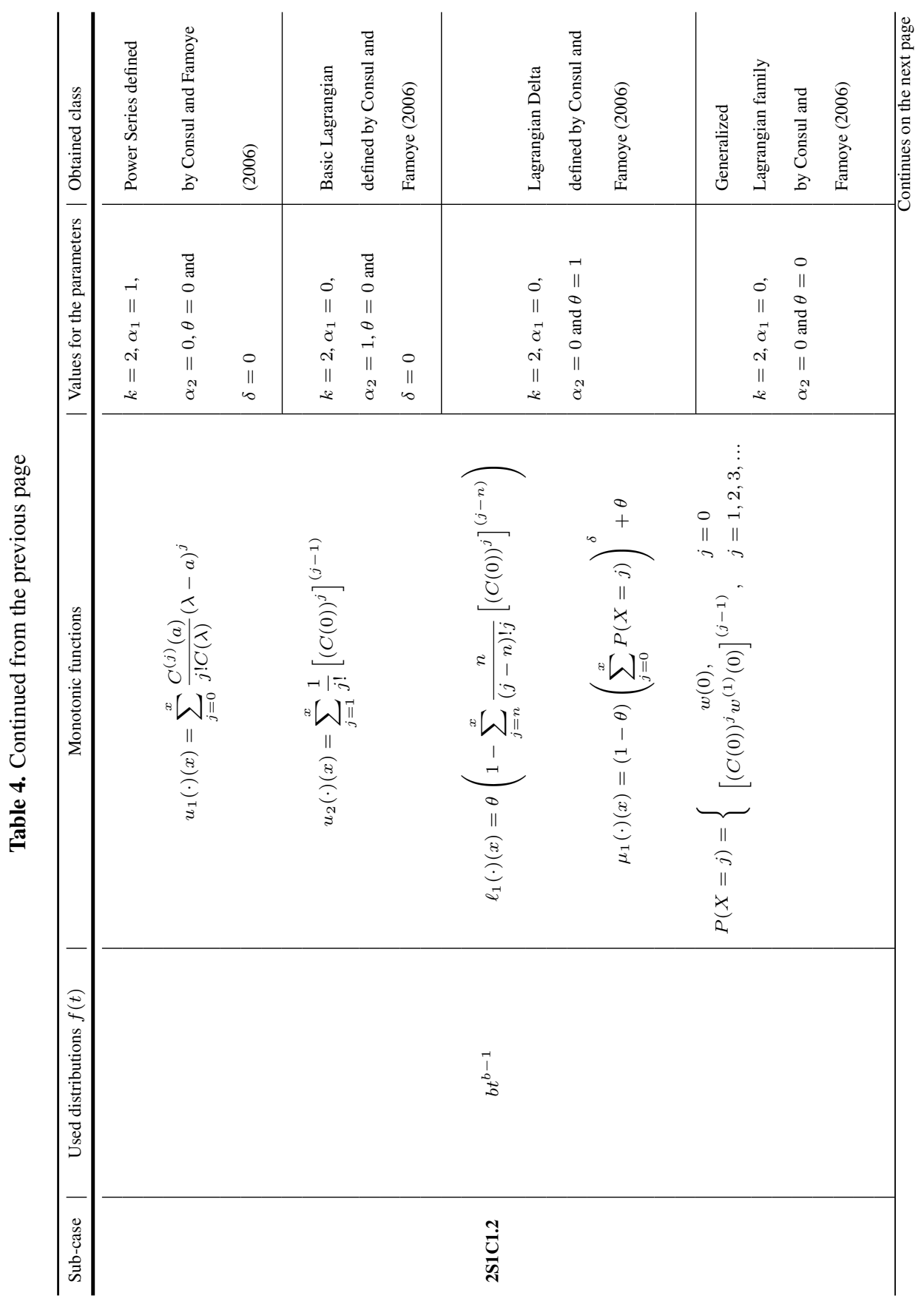




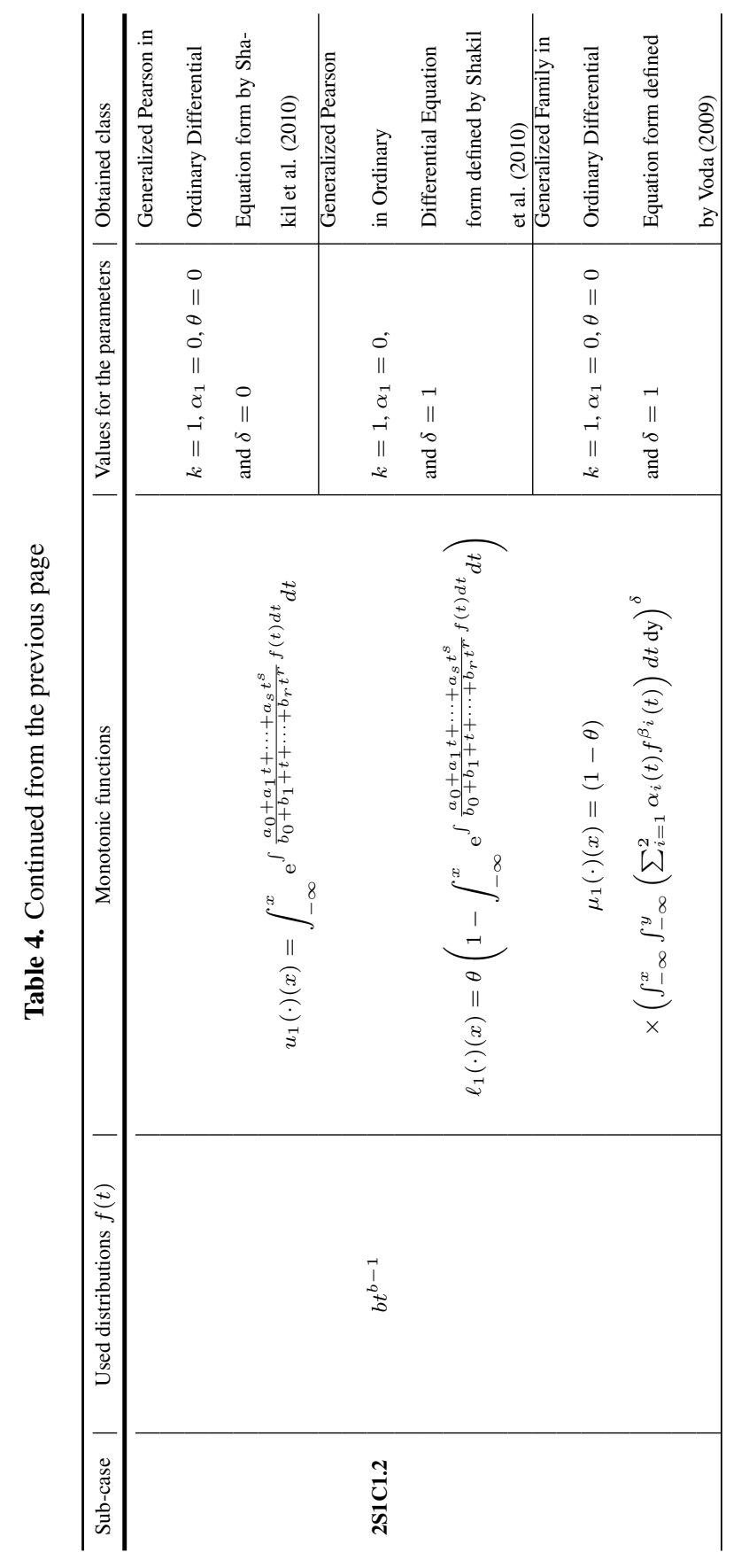




\section{References}

[1] Alzaatreh, A., Lee, C. and Famoye F. A new method for generating families of continuous distributions, Metron 71, 63-79, 2013.

[2] Brito, C.R., Gomes-Silva, F., Rêgo, L.C. and Oliveira, W.R. A new class of gamma distribution, Acta Sci-Technol 39, 79-89, 2017.

[3] Cordeiro, G.M., Alizadeh, M. and Silva, R.B. A New Wider Family of Continuous Models: The Extended Cordeiro and de Castro Family, Hacet J Math Stat, 2017, In press.

[4] Cordeiro, G.M. and de Castro, M. A new family of generalized distributions, J Stat Comput Simul 81, 883-898, 2011.

[5] Consul, P.C. and Famoye, F. Lagrangian Probability Distributions, Birkhäuser Boston, Boston, 2006.

[6] Eugene, N., Lee, C. and Famoye, F. Beta-normal distribution and its application, Commun Stat Theor Meth 31, 497-512, 2002.

[7] Gomes-Silva, F., Percontini, A., Brito, E., Ramos, M.W.A., Silva, R.V. and Cordeiro, G.M. The odd Lindley-G family of distributions, Austrian J Stat 49, 57-79,2017.

[8] Jayakumar, K. and Mathew, T. On a generalization to Marshall-Olkin scheme and its application to Burr type XII distribution, Stat Pap 49, 421-439, 2008.

[9] Lee, C., Famoye, F. and Alzaatreh, A. Methods for generating families of univariate continuous distributions in the recent decades, WIREs Comput Stat 5, 219-238, 2013.

[10] Marshall, A.W. and Olkin, I.A. A new method for adding a parameter to a family of distributions with application to the exponential and weibull families, Biometrika 84, 641-652, 1997.

[11] McDonald, J.B. Some generalized functions for the size distribution of income, Econometrica 52, 647-663, 1984.

[12] Mudholkar, G.S., Srivastava, D. K. and Freimer, M. The Exponentiated Weibull Family: A Reanalysis of the Bus-Motor-Failure Data. Technometrics 37, 436-445, 1995.

[13] Percontini, A. New Extended Lifetime Distributions, Ph. D. thesis in Computational mathematics, Federal University of Pernambuco, Brazil, 2014.

[14] Percontini, A., Cordeiro, G.M. and Bourguignon, M. The G-Negative Binomial Family: General Properties and Applications, Adv Appl Stat 35, 127-160, 2013.

[15] Percontini, A., Blas, B. and Cordeiro, G.M. The beta weibull poisson distribution, Chil J Stat 4 3-26, 2013.

[16] Pescim, R.R., Cordeiro, G.M., Demétrio, C.G.B. and Nadarajah, S. The new class of Kummer beta generalized distributions, SORT 36, 153-180, 2012.

[17] Ramos, M.W.A., Marinho, P.R.D., Cordeiro, G.M., Silva, R.V. and Hamedani, G. The kumaraswamy-G Poisson family of distributions, J Stat Theory Appl 14, 222-239, 2015.

[18] Silva, R.B., Bourguignon, M., Dias, C.R.B. and Cordeiro, G.M. The compound class of extended Weibull power series distributions, CSDA 58, 352-367, 2013.

[19] Silva, R.V., Gomes-Silva, F., Ramos, M.W.A., Cordeiro, G.M., Marinho, P.R.D. and Andrade, T.A.N. The Exponentiated Kumaraswamy-G Class: General Properties and Application, Rev Colomb Estad 2019, Forthcoming.

[20] Shakil, M., Kibria, B.M. and Singh, J.N. A new family of distributions based on the generalized Pearson differential equation with some applications, Aust J Stat 39, 259-258, 2010.

[21] Tahir, M.H. and Cordeiro, G.M. Compounding of distributions: a survey and new generalized classes, JSDA 3, 1-35, 2016.

[22] Tahir, M. H. and Nadarajah, S. Parameter induction in continuous univariate distributions: Well established $G$ families, An Acad Bras Cienc 87, 539-568, 2015.

[23] Voda, V. G. A method constructing density functions: the case of a generalized Rayleigh variable, Appl Math 54, 417-431, 2009.

[24] Zografos, K. and Balakrishnan, N. On the families of beta-and generalized gamma-generated distribution and associated inference, Stat Met 6, 344-362, 2009. 